\title{
RNA sequencing of blood in coronary artery disease: involvement of regulatory $T$ cell imbalance
}

Timothy A. McCaffrey ${ }^{1,4,6,10^{*}}$ D , lan Toma ${ }^{1,7,10}$, Zhaoquing Yang ${ }^{1}$, Richard Katz ${ }^{2}$, Jonathan Reiner ${ }^{2}$, Ramesh Mazhari ${ }^{2}$, Palak Shah' ${ }^{9}$, Michael Tackett ${ }^{3}$, Dan Jones ${ }^{3}$, Tisha Jepson 3,4,10, Zachary Falk', Richard Wargodsky' ${ }^{1}$ Dmitry Shtakalo ${ }^{8}$, Denis Antonets ${ }^{8}$, Justin Ertle', Ju H. Kim², Yinglei Lai ${ }^{5}$, Zeynep Arslan', Emily Aledort ${ }^{1}$, Maha Alfaraidy ${ }^{1}$ and Georges St. Laurent II $^{4}$

\begin{abstract}
Background: Cardiovascular disease had a global prevalence of 523 million cases and 18.6 million deaths in 2019. The current standard for diagnosing coronary artery disease (CAD) is coronary angiography. Surprisingly, despite well-established clinical indications, up to $40 \%$ of the one million invasive cardiac catheterizations return a result of 'no blockage.' The present studies employed RNA sequencing of whole blood to identify an RNA signature in patients with angiographically confirmed CAD.
\end{abstract}

Methods: Whole blood RNA was depleted of ribosomal RNA (rRNA) and analyzed by single-molecule sequencing of RNA (RNAseq) to identify transcripts associated with CAD (TRACs) in a discovery group of 96 patients presenting for elective coronary catheterization. The resulting transcript counts were compared between groups to identify differentially expressed genes (DEGs).

Results: Surprisingly, $98 \%$ of DEGs/TRACs were down-regulated 1.7-fold in patients with mild to severe CAD (> 20\% stenosis). The TRACs were independent of comorbid risk factors for CAD, such as sex, hypertension, and smoking. Bioinformatic analysis identified an enrichment in transcripts such as FoxP1, ICOSLG, IKZF4/EOS, SMYD3, TRIM28, and TCF3/E2A that are likely markers of regulatory T cells (Treg), consistent with known reductions in Tregs in CAD. A validation cohort of 80 patients confirmed the overall pattern (92\% down-regulation) and supported many of the Treg-related changes. TRACs were enriched for transcripts associated with stress granules, which sequester RNAs, and ciliary and synaptic transcripts, possibly consistent with changes in the immune synapse of developing $T$ cells.

Conclusions: These studies identify a novel mRNA signature of a Treg-like defect in CAD patients and provides a blueprint for a diagnostic test for CAD. The pattern of changes is consistent with stress-related changes in the maturation of $T$ and Treg cells, possibly due to changes in the immune synapse.

Keywords: Atherosclerosis, Transcriptome, RNA sequencing, Regulatory T cells, Treg, FoxP1, FoxP3, Biomarker, Coronary artery disease, Stress granules, Cilia, Immune synapse

*Correspondence: mcc@gwu.edu

${ }^{1}$ Division of Genomic Medicine, Department of Medicine, The George Washington Medical Center, The George Washington University, 2300 I Street NW, Ross Hall 443A, Washington, DC 20037, USA

Full list of author information is available at the end of the article

\section{Background}

There are more than a million heart attacks each year, and 2200 Americans die of cardiovascular disease each day, about one person every $40 \mathrm{~s}$ [1]. Outward symptoms of coronary artery disease (CAD) are chest pain, 
typically radiating down the left arm, and shortness of breath upon exertion. However, chest pain and dyspnea alone are not particularly specific warning signs. In a prospective analysis of patients presenting with chest pain, ultimately, many cases were determined to be musculoskeletal $(20 \%)$ or gastroesophageal reflux disease (GERD) (13\%), while CAD was diagnosed in only $11 \%$ of cases, and the remaining cases were either pulmonary [2], neurological, or idiopathic [3]. The Framingham risk factors of advanced age, male sex, elevated cholesterol, smoking, and hypertension, are good predictors of long term risk ( $30 \mathrm{yr}$. risk, $\mathrm{C}$ statistic $=0.803$ ) [4], but they are far less accurate in acute clinical settings at determining whether a person has CAD or not (C statistic $=0.667$, where 0.5 is random chance) [5]. Thus, there is a tremendous need for improvement in the diagnosis of CAD. From the more than one million cardiac catheterizations yearly, 622,000 result in interventions such as stent placement [6]. Despite the presence of CAD symptoms and other clinical tests suggestive of CAD, $20-40 \%$ of angiograms do not detect any occluded arteries [5, 7-10]. The American College of Cardiology's Registry, covering 398,978 patients, identified $39.2 \%$ of patients undergoing invasive coronary angiography (ICA) as having less than 20\% stenosis [5]. Thus, reliable blood-based biomarkers of CAD would have the potential to reduce the number of cardiac catheterizations on relatively low risk individuals.

Several prior microarray studies suggested that there is an RNA signature in blood associated with CAD [11-15]. However, the agreement between these studies on exactly which transcripts are modulated is quite low. Such discrepancies could have several explanations, but likely arise from cross-hybridization noise created by highly abundant signals, such as globins, which can overwhelm true signals in microarrays [16], and likely mask changes of low magnitude, or larger changes in a small subset of cells. Thus, the present studies employed a more advanced, single-molecule RNA sequencing (RNAseq) methodology to identify diagnostic transcripts associated with CAD (TRACs). Using RNAseq of whole blood RNA, a novel pattern of gene expression changes was identified that is associated with the presence of CAD, but essentially unrelated to other known risks for CAD. This subset of TRACs is consistent with extensive accumulating evidence for a role of regulatory $\mathrm{T}$ cell (Treg) dysfunction as an important component in the etiology of CAD.

\section{Methods}

\section{Experimental design}

The studies take advantage of the fact that up to $40 \%$ of patients that undergo invasive coronary angiography
(ICA) actually do not have meaningful coronary blockage. The TRACs were identified by comparing the mRNA expression pattern of patients with CAD versus those without CAD. The strength of this model is that blood was taken prior to the catheterization, and the outcome of the angiography becomes known within hours, which provides an ideal learning environment for designing a transcriptome-based test. After the coronary angiograms were digitally interpreted by an attending physician, the patients were divided into 3 groups, $\leq 20 \%$ stenosis (LOW CAD), $>20 \%$ but $<70 \%$ stenosis of any vessel (MID CAD), and $\geq 70 \%$ stenosis of any artery (CAD). For power and simplicity, initial analyses compared LOW CAD $(<20 \%$ stenosis $)$ to MID+ (> 20\% stenosis).

\section{Patients}

\section{Discovery cohort}

Patients presenting for non-emergent complaints of typical or atypical chest pain, exertional dyspnea, or other symptoms suggestive of CAD provided written, informed consent for participation in this study under a protocol approved by the George Washington University IRB. Patients with heart failure, non-ST segment elevation MI, and ST segment elevation MI (STEMI) were excluded from the study. The design of the study is shown schematically in Fig. 1. Patients admitted for cardiac catheterization had three Tempus blood RNA tubes collected by peripheral venipuncture or an indwelling catheter. After blood sampling, these studies were purely observational and did not alter in any way the patient's clinical course. All relevant clinical data, including a complete blood count $(\mathrm{CBC})$, was captured for comparison to the transcriptomic studies. From an initial enrollment of 113 patients, 96 patients had complete clinical and RNA sequencing data for further analysis.

\section{Validation cohort}

An independent group of patients were consented at INOVA Fairfax Hospital (Fairfax, Virginia) who were likewise undergoing routine, elective ICA for evaluation of suspected CAD. A total of 80 patients had sufficiently complete clinical and RNAseq data for further analysis.

\section{Clinical prediction model}

Prior to ICA in the Discovery cohort, cardiac medical histories were examined by their attending cardiologists to determine CAD risk factors, as defined by the 2013 ACC/AHA Guidelines on the Assessment of Cardiovascular Risk [17]. Hypertension was defined as a history of untreated blood pressure $\geq 140 / 90 \mathrm{mmHg}$ 


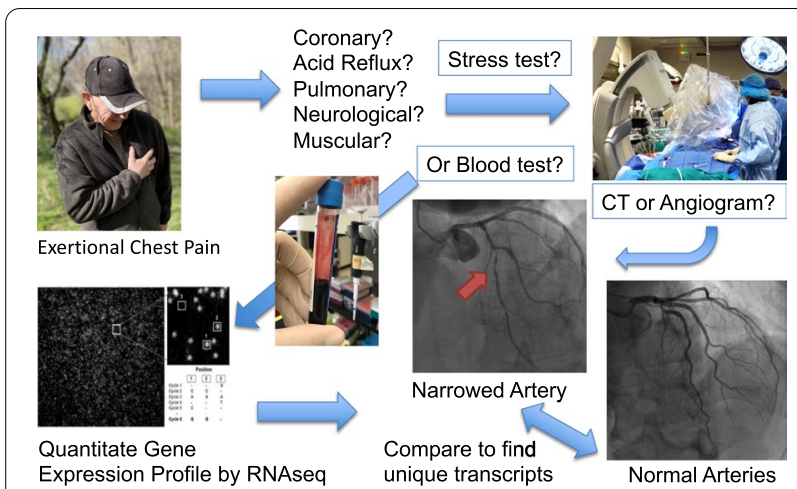

Fig. 1 Schematic of study design. Patients presenting for elective invasive coronary angiography (ICA) due to suspicion of CAD were consented to determine whether RNA transcripts in blood could serve as biomarkers for CAD. Typically, patients reported chest pain or shortness of breath upon exertion. The results of the angiogram divided the patients into groups with little to no coronary blockage $(<20 \%$, LOW CAD), or patients in which coronary blockage was detected (> 20\%, MID+ CAD). The blood from the patients was frozen in Tempus blood RNA preservative, thawed, extracted for RNA, depleted of residual genomic DNA and ribosomal RNA, and genome-wide RNA transcript counting was performed by RNAseq. The two groups were compared to identify transcripts unique to the CAD patients. Images were created by the authors

and/or treatment with anti-hypertensive medications. Diabetes mellitus was defined by fasting glucose of $\geq 126 \mathrm{mg} / \mathrm{dl}$ and/or use of insulin or oral hypoglycemic agents. Dyslipidemia was defined according to National Cholesterol Education Program Adult Treatment Panel III guidelines or by treatment with lipid lowering medication. Current smoking status was defined by active smoking within 3 months of presentation. A family history of CAD was defined as MI or cardiac death in a first-degree relative.

Chest pain was classified according to standard criteria for angina pectoris as described [18]. Typical angina includes substernal, jaw, and/or arm pain upon exertion, and which resolves within $15 \mathrm{~min}$ of rest and/or use of nitroglycerin. Atypical angina involves 2 of these symptoms, and patients with non-cardiac chest pain experienced 1 or none of these symptoms. Dyspneic patients were classified as having symptoms of a typical angina.

From these clinical parameters, risk points were accumulated based on age, sex, hypertension, diabetes, symptom type, family history, and smoking status, and then compared to an ordinal risk model to predict likelihood of CAD [18].

\section{Transcriptome profiling}

RNA processing

RNA was purified from Tempus stabilized frozen $\left(-80{ }^{\circ} \mathrm{C}\right)$ peripheral blood samples using Tempus Spin
RNA Isolation Kit (ThermoFisher Scientific) according to the manufacturer's protocol. After rigorous in-solution treatment with 4 Units of DNAse (Turbo DNA-free Kit, Ambion), the typical nucleic acid yield from $2.5 \mathrm{ml}$ Tempus blood tubes averaged $\sim 5 \mu \mathrm{g}$, with an RNA integrity (RIN) score $>8$ (10 is maximal) on Agilent 2100 Bioanalyzer. A fixed amount (4.5 ug) of the DNAsed total RNA was depleted of ribosomal RNA (rRNA) by Ribo-Zero rRNA Removal Kit (Illumina), then concentrated with an RNeasy MinElute column (Qiagen), resulting in $~ 500 \mathrm{ng}$ RNA for sequencing.

\section{RNA sequencing}

For RNAseq, 100 ng of rRNA-depleted RNA was fragmented and analyzed on a Heliscope true single molecule sequencer (tSMS, SeqLL, Inc.). The raw reads, typically 40 million at $38 \mathrm{bp}$ average length, were then computationally aligned to the human genome using the Helisphere indexDPgenomic aligner [19]. The number of reads that align to each transcript was counted and then corrected for transcript length and differences in total reads obtained per patient. The raw read count was adjusted by the size of the transcript so that long transcripts do not appear more highly expressed than short transcripts, and by the number of total reads per sample to produce "Reads Per Kilobase of transcript, per Million mapped" (RPKM) counts. Thus, RPKM corrects the expression level between samples that have different absolute numbers of reads. RPKM levels were imported into GeneSpring GX14 suite, without additional normalization, to identify transcripts that differ between CAD groups (TRACs). Differentially expressed genes (DEGs) were identified by filtering low expression genes, and then applying a combined p-value/fold change thresholds using a Volcano plot, and Analysis of Variance (ANOV) in Genespring.

\section{Comparison of blood RNA preservation/isolation methods by droplet digital PCR (ddPCR)}

To determine whether TRACs were affected by the type of blood RNA preservation method, three Tempus and three Paxgene tubes were drawn from the same subjects at the same time. The samples were isolated according to manufacturer's protocols, with the exception that the Paxgene samples were not DNAsed on column, instead using the Turbo DNA-free kits (Ambion) on total RNA as a separate step, so as to be comparable with RNA isolated from Tempus tubes. After DNAse, the samples were repurified with RNeasy MinElute kit (Qiagen) and cDNA was reverse transcribed from $500 \mathrm{ng}$ of RNA using the iScript cDNA synthesis kit (Bio-Rad). The synthesized cDNA was diluted $15 \times$ to $7 \mathrm{ng} / \mu \mathrm{l}$ and $5 \mu \mathrm{l}$ per reaction were used in ddPCR combined with $15 \mu \mathrm{l}$ QX200 
EvaGreen ddPCR Supermix (Bio-Rad) containing $1 \mu \mathrm{l}$ of 2.5 pmol primers diluted from the original stock of 100 $\mathrm{uM}(\mathrm{pmol} / \mathrm{uL})$. The ddPCR droplets were generated with Automated Droplet Generator and signals were amplified using the standard ddPCR protocols on a C100 Thermal Cycler (Bio-Rad).

The Paxgene versus Tempus cDNAs were then analyzed with a set of 'invariant' PCR targets (beta-actin (ACTB) and alpha-tubulin (TBA1)), selected TRACs (DGKA, DLG1, ICOSLG, IKZF4, SMYD3, TCF3, TRIM28), and selected targets unrelated to TRACs (DEFA3, SELL, SOD2, IL12A). The abundance of each transcript was expressed as a ratio of the copies $/ 20 \mu \mathrm{l}$ per target in Tempus vs Paxgene samples $(\mathrm{n}=4$ samples from 3 subjects).

\section{Results}

Clinical parameters

Discovery cohort

From a total of 112 patients enrolled, 96 patients had sufficient RNA quantity and quality, and adequate RNA read depth for further analysis. The clinical parameters of those 96 patients were generally comparable between the LOW and MID+ CAD groups. After correction for multiple testing, there were no significant differences correlated with age, ethnicity, sex, BMI, current smoking, hypertension, dyslipidemia, diabetes, or aspirin use (Table 1). However, there was a trend for the group of LOW CAD patients to be somewhat younger (57.5 yr LOW vs 62.5 yr MID+), and with fewer males (43.8\% male LOW vs $56.2 \%$ male MID+). To consider any possible confounding variables, we performed separate comparative analysis of all major clinical parameters as regulators of transcript profiles in blood against selected TRACs.

\section{Validation cohort}

Patients were recruited from an ongoing cohort examining the relationship between DNA variations and CAD. A total of 80 patients had acceptable RNAseq data for further analysis. This suburban Virginia cohort had somewhat different demographics, with mainly the minority composition dropping from more than $50 \%$ in Discovery group to less than $20 \%$ in the validation group, as shown in Table 2.

\section{Analytical parameters}

The yield of RNA and the number of reads per patient did not vary significantly, when multiple testing was considered (Table 1). Of the 112 samples submitted for sequencing, 16 were excluded due to low yield from RNA purification or ribosomal depletion, inefficient cDNA synthesis, or low yield of usable reads from RNAseq.
There was a trend that was not statistically significant when corrected for multiple testing that the LOW CAD patients had slightly higher RNA yield $(6.09 \mu \mathrm{g} /$ tube LOW, vs. $4.82 \mu \mathrm{g} /$ tube MID $+\mathrm{CAD}, \mathrm{p}=0.07$ uncorrected) and higher read depth ( $\mathrm{p}=0.02$ uncorrected) on RNAseq. Thus, these were considered as possible confounds in subsequent analysis.

\section{Sources of variation in RNA yield}

Patient blood samples collected with either Paxgene or Tempus RNA preservation tubes show a surprisingly large variation in the RNA yield, with Tempus generally producing higher total RNA yield [20]. The total nucleic acid yield from Tempus-preserved samples ranged from 0.6 to $35.0 \mu \mathrm{g} /$ tube whole blood, with a mean of $10.6 \mu \mathrm{g}$ per tube of blood, with post-DNAse and MinElute cleanup yield of $\sim 5 \mu \mathrm{g}$ RNA per tube (Table 1). The correlations between total nucleic acid yield and any single blood cell count parameter were quite weak, with only a modest correlation to absolute lymphocyte count $\left(r=0.55\right.$ with $\mathrm{N}=112, \mathrm{R}^{2}=0.31$ ) (Additional file 1 : Fig. S1). While one outlier with a lymphocyte count of $12 \mathrm{~K} / \mu \mathrm{l}$ yielding $35 \mu \mathrm{g}$ of RNA seems to drive this correlation, the correlation remains modestly positive even when that patient is omitted $(r=0.45 \mathrm{w} / \mathrm{o})$. Thus, the lymphocyte count is the major factor in RNA yield, but accounts for only about $30 \%$ of the variability.

\section{Whole blood RNA biomarkers}

The RNAseq data was subjected to minimal normalization, using only the raw RPKM data for analysis. When sequencing was completed, the GRCh37/hg19 assembly was the most fully annotated in our lab. The RNAseq reads were aligned, and then parsed and counted against the 161,038 transcripts in hg19. Transcripts with very low-level expression were filtered by requiring RPKM $>0.01$ in $70 \%$ of the samples of at least one group, which had a minimal impact on the number of included transcripts $(157,943)$. Dividing the samples by CAD level $\leq 20 \%$ (LOW, $n=48$ ) versus $>20 \%$ (MID+, $\mathrm{n}=48$ ) and averaging across patients yielded the geometric mean expression per group per transcript, as shown in Fig. 2. Remarkably, without any normalization per sample beyond RPKM, the RNAseq data shows excellent linearity over $23 \log _{2}$ orders of magnitude, with the highest level of gene expression observed for hemoglobin $\mathrm{B}$, at an average of RPKM of $\sim 65 \mathrm{~K}$ (16 in $\log _{2}$ scale) in both groups. Compared to typical microarray data, the RNAseq shows less noticeable increases in variability at low levels of gene expression, and no detectable saturation of the signal at very high gene expression. 
Table 1 Patient demographics: discovery cohort

\begin{tabular}{|c|c|c|c|c|c|}
\hline & \multicolumn{5}{|c|}{ Coronary artery stenosis } \\
\hline & \multicolumn{2}{|l|}{ Low } & \multicolumn{2}{|l|}{ MID+ } & \multirow{2}{*}{$\begin{array}{l}\text { P value* } \\
\text { uncorrected }\end{array}$} \\
\hline & Mean & S.E.M & Mean & S.E.M & \\
\hline N per group & 48 & & 48 & & \\
\hline Age (years) & 57.5 & 1.49 & 62.5 & 1.41 & $0.02^{*}$ \\
\hline Race (\% minority) & 62.5 & & 45.8 & & 0.10 \\
\hline Sex (\% male) & 45.8 & & 56.2 & & 0.31 \\
\hline BMI & 34.7 & 1.32 & 31.4 & 0.97 & $0.05^{*}$ \\
\hline Current smoker (\%) & 8.3 & & 14.6 & & 0.34 \\
\hline Hypertension (\%) & 70.8 & & 75.0 & & 0.86 \\
\hline Systolic BP & 135.85 & 3.02 & 137.05 & 3.80 & 0.81 \\
\hline Diastolic BP & 72.67 & 1.66 & 72.67 & 1.83 & 1.00 \\
\hline Dyslipidemia (\%) & 58.3 & & 70.8 & & 0.28 \\
\hline Total Chol. (mg/dL) & 180.50 & 4.80 & 167.09 & 4.91 & 0.62 \\
\hline LDL Chol. (mg/dL) & 109.00 & 4.49 & 95.64 & 4.74 & 0.61 \\
\hline VLDL Chol. (mg/dL) & 13.00 & 0.61 & 23.45 & 1.60 & 0.23 \\
\hline HDL Chol & 51.00 & 0.61 & 47.82 & 1.40 & 0.67 \\
\hline Tri-glycerides & 66.00 & 3.06 & 117.45 & 7.97 & 0.23 \\
\hline Creatine kinase-(U/L) & 152.00 & 19.60 & 172.75 & 19.78 & 0.87 \\
\hline Diabetes (\%) & 35.4 & & 33.3 & & 0.83 \\
\hline Aspirin (\%) & 52.1 & & 62.5 & & 0.21 \\
\hline $\mathrm{PTT}(\mathrm{s})$ & 29.77 & 0.60 & 30.96 & 0.67 & 0.19 \\
\hline $\mathrm{PT}(\mathrm{s})$ & 12.97 & 0.24 & 13.13 & 0.41 & 0.74 \\
\hline INR & 0.99 & 0.02 & 0.96 & 0.01 & 0.26 \\
\hline $\mathrm{WBC}\left(\times 10^{3} / \mathrm{uL}\right)$ & 6.99 & 0.35 & 6.92 & 0.36 & 0.89 \\
\hline $\mathrm{RBC}\left(\times 10^{6} / \mathrm{uL}\right)$ & 4.63 & 0.07 & 4.37 & 0.07 & $0.02^{*}$ \\
\hline Hemoglobin (g/dL) & 13.19 & 0.17 & 13.16 & 0.24 & 0.91 \\
\hline Hematocrit (\%) & 39.79 & 0.48 & 39.02 & 0.62 & 0.33 \\
\hline $\mathrm{MCV}(\mathrm{fL})$ & 85.94 & 0.75 & 89.01 & 0.69 & $0.01^{*}$ \\
\hline $\mathrm{MCH}(\mathrm{pg})$ & 28.45 & 0.30 & 30.00 & 0.26 & $0.00^{*}$ \\
\hline $\mathrm{MCHC}(\mathrm{g} / \mathrm{dL})$ & 33.06 & 0.17 & 33.70 & 0.18 & $0.02^{*}$ \\
\hline RDW (\%) & 14.16 & 0.25 & 13.03 & 0.27 & $0.01^{*}$ \\
\hline Platelet Count $\left(\times 10^{3} / \mathrm{uL}\right)$ & 249.81 & 8.08 & 228.98 & 8.84 & 0.09 \\
\hline MPV (fL) & 10.63 & 0.13 & 10.57 & 0.14 & 0.76 \\
\hline Seg. neutrophils (\%) & 56.34 & 1.83 & 62.30 & 1.59 & $0.03^{*}$ \\
\hline Lymphocyte \% & 32.76 & 1.74 & 26.21 & 1.38 & $0.01^{*}$ \\
\hline Eosinophil \% & 2.34 & 0.19 & 2.33 & 0.26 & 0.96 \\
\hline Basophil \% & 0.40 & 0.07 & 0.24 & 0.07 & 0.17 \\
\hline Abs. seg. neutrophils $\left(\times 10^{3} / \mathrm{uL}\right)$ & 4.08 & 0.24 & 4.53 & 0.32 & 0.31 \\
\hline Abs. lymphocytes (× 103/uL) & 2.42 & 0.25 & 1.72 & 0.09 & $0.02^{*}$ \\
\hline Abs. eosinophils (× 103/uL) & 0.16 & 0.01 & 0.16 & 0.02 & 0.95 \\
\hline Absolute basophils ( $\left.\times 10^{3} / \mathrm{uL}\right)$ & 0.04 & 0.00 & 0.03 & 0.00 & 0.22 \\
\hline Auto monocyte \% & 7.53 & 0.34 & 8.35 & 0.39 & 0.15 \\
\hline Auto monocyte \# (× 103/uL) & 0.53 & 0.03 & 0.56 & 0.03 & 0.51 \\
\hline Immature granulocytes \% & 0.24 & 0.02 & 0.25 & 0.02 & 0.62 \\
\hline RNA yield (ng/ul) 50 ul total & 121.72 & 11.1113 & 96.48 & 7.50 & 0.07 \\
\hline Yield (ug/2.5 ml tube) & 6.09 & & 4.82 & & \\
\hline Total RNAseq reads & $6.08 \mathrm{E}+07$ & $4,189,732$ & $5.35 \mathrm{E}+07$ & $3,691,059$ & 0.19 \\
\hline Filtered reads & $2.44 \mathrm{E}+07$ & $1,876,258$ & $2.02 \mathrm{E}+07$ & $1,522,361$ & 0.09 \\
\hline Aligned reads (informative) & $8.75 E+06$ & 773,714 & $6.65 E+06$ & $474,229.5$ & $0.02^{*}$ \\
\hline
\end{tabular}


Table 2 Patient demographics: validation cohort

\begin{tabular}{|c|c|c|c|c|c|}
\hline & \multicolumn{5}{|c|}{ Coronary artery stenosis } \\
\hline & \multicolumn{2}{|l|}{ Low } & \multicolumn{2}{|l|}{ MID+ } & \multirow{2}{*}{$\begin{array}{l}\text { P value } \\
\text { uncorrected }\end{array}$} \\
\hline & Mean & S.E.M & Mean & S.E.M & \\
\hline N per group & 37 & & 43 & & \\
\hline Sex (\% male) & $62.16 \%$ & & $51.16 \%$ & & 0.32 \\
\hline Age (years) & 62.89 & 1.74 & 67.61 & 1.79 & 0.06 \\
\hline Race (\% minority) & $16.22 \%$ & & $9.30 \%$ & & 0.99 \\
\hline Hispanic (\%) & $5.41 \%$ & & $2.33 \%$ & & 0.47 \\
\hline Height (cm) & 173.54 & 2.12 & 170.39 & 1.57 & 0.23 \\
\hline Weight (kg) & 96.11 & 4.69 & 86.59 & 3.25 & 0.09 \\
\hline BMI & 32.01 & 1.57 & 29.83 & 1.04 & 0.24 \\
\hline SBP & 124.62 & 2.58 & 127.42 & 3.02 & 0.49 \\
\hline DBP & 70.19 & 1.67 & 68.58 & 1.40 & 0.46 \\
\hline MAP & 88.35 & 1.67 & 88.23 & 1.68 & 0.96 \\
\hline$H R$ & 69.68 & 1.95 & 71.00 & 2.17 & 0.66 \\
\hline Heart Rate & 69.68 & 1.95 & 71.00 & 2.17 & 0.66 \\
\hline EF \% & 54.70 & 1.67 & 58.16 & 1.51 & 0.13 \\
\hline Dyslipidemia & $43.24 \%$ & & $65.12 \%$ & & $0.05^{*}$ \\
\hline Hypertension & $67.57 \%$ & & $67.44 \%$ & & 0.99 \\
\hline Diabetes mellitus & $21.62 \%$ & & $23.26 \%$ & & 0.86 \\
\hline Smoking & $5.41 \%$ & & $11.63 \%$ & & 0.33 \\
\hline Aspirin & $48.65 \%$ & & $76.74 \%$ & & $0.01^{*}$ \\
\hline Creatinine & 0.96 & 0.04 & 0.98 & 0.04 & 0.69 \\
\hline \%Stenosis & 1.57 & 0.48 & 66.79 & 3.97 & $6.50 E-25^{*}$ \\
\hline RNA yield (ng/ul) & 81.36 & 2.34 & 84.54 & 2.15 & 0.32 \\
\hline Total RNA/tube (ng) & 7322.59 & & 7608.50 & & \\
\hline
\end{tabular}

\section{Identification of differentially expressed RNA biomarkers for CAD}

The 96 samples in the Discovery cohort were divided into LOW CAD $(<20 \%$ stenosis, $\mathrm{N}=48)$ versus MID $+\mathrm{CAD}$ ( $>20 \%$ stenosis, $N=48$ ). The transcripts were filtered to exclude low expression transcripts $(<0.01 \mathrm{RPKM})$ and then compared by ANOV $(\mathrm{p}<0.001)$ to identify a small set of differentially expressed genes (DEGs). This initial filtering identified 198 transcripts that include the 59 transcripts highlighted in BLACK in Fig. 2 and detailed in Additional file 2: Table S1.

By filtering the 198 transcripts for those which had a $>20$ th percentile absolute level of expression (RPKM percentile) in both groups, the list was narrowed to 96 transcripts with higher absolute expression. Of those 96 transcripts, 51 showed a greater than 1.4-fold decrease in the MID + CAD group, which became the parent list for identifying smaller sets of CAD-related transcripts. This combined fold-change/t-test strategy has been established in large, multicenter control studies using spiked samples as a reliable approach to identify true differences [21].

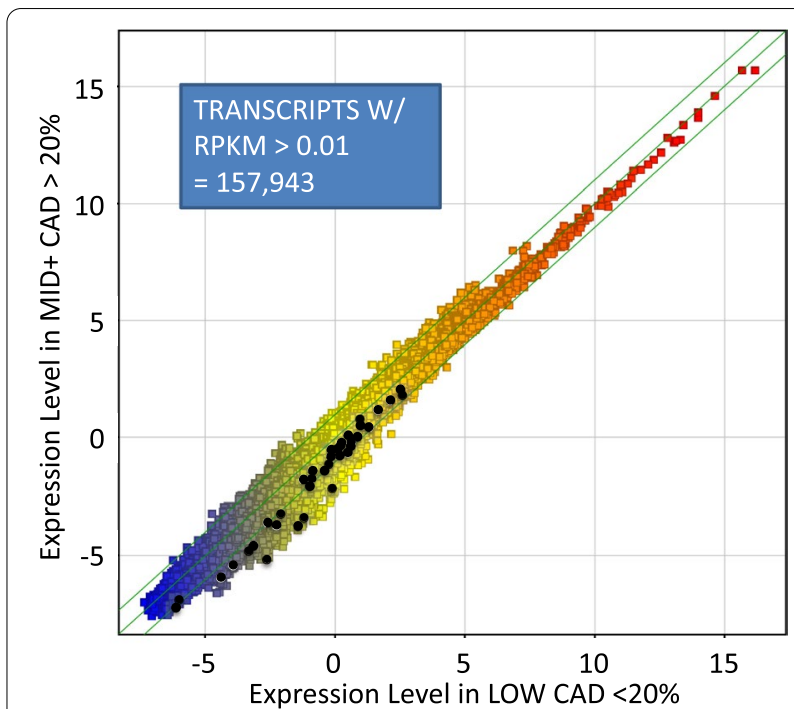

Fig. 2 Genome-wide transcript profiling by RNAseq. A total of 96 patients with angiographic results were analyzed by RNAseq of whole blood RNA depleted of ribosomal sequences. The short reads were aligned to the human transcriptome (hg19) and counted per transcript. The raw read counts $(R)$ were normalized only by (Per) the length of the transcript $(K)$ and the total number of reads obtained per patient in millions (M) to yield RPKM. The RPKM is expressed on a $\log _{2}$ scale and averaged across all patients in the LOW CAD group ( $n=48, X$ axis) versus patients in the MID+ CAD group ( $n=48, Y$ axis). Each point represents one transcript where the RPKM was $>0.01$ RPKM in $70 \%$ of samples in at least one group $(157,943$ transcripts). Black points represent a set of transcripts identified as differentially expressed between the 2 groups by a statistical analysis of fold-change and t-test probability ( $p<0.001$ uncorrected, and fold change $>1.5$ ) resulting in 59 transcripts (49 unique, non-redundant)

\section{Comparison of TRACs relative to transcripts related to clinical risk factors}

Because CAD has several known risk factors, such as hypertension, smoking, and dyslipidemia, the relationship of TRACs to these other parameters was determined. While not strictly statistically significant, the demographic analysis suggested that the LOW CAD group tended to be younger, heavier, and more female. For comparison purposes, classifying the 96 patients by sex (48 M, $48 \mathrm{~F}$ ) irrespective of CAD status, and using a combined filter for $>$ threefold change and $\mathrm{p}<0.05$ (uncorrected), the analysis identified 84 transcripts that were 'sex-specific' (Additional file 2: Table S2). This included transcripts from the X (XIST) and Y chromosomes, and yielded an PLSD prediction model that was 97\% accurate (100\% accurate for males, $95 \%$ for females), simply confirming that the RNAseq data can readily detect obvious biological differences.

Using a similar approach, RNA biomarkers lists were constructed for age (young <60 YO), hypertension, dyslipidemia, BMI, smoking, diabetes, and aspirin use. 
While each biomarker list showed interesting changes in RNA expression levels, there was very minor overlap with the TRAC list (Fig. 3). Five transcripts (ABCF2, CHST10, FAM129C, MAST4, TEX41) were sensitive to CAD and $\mathrm{BMI}$, even though, to minimize confounding, the BMI list was derived from LOW CAD patients only. SMYD3 was identified as sensitive to the age of the subject, albeit with an alternative transcript ID compared to the TRAC list. However, the direction of the change, whereby SMYD3 increased with age, is opposite to the change expected on the basis of the age of the patients with CAD, and thus, age is somewhat offsetting the CAD effect on SMYD3. Aspirin use was more common in the MID+ group, but its correlation with TRACs was statistically nonsignificant. Two transcripts were identified that were both TRACs and aspirin-sensitive: CHST10 (decreased only at $81 \mathrm{mg} /$ day dose) and NT5C3B (decreased only at $325 \mathrm{mg}$ /day dose). In general, however, there was little to no evidence that the TRACs are related to other known clinical correlates of CAD.

\section{Relationship of TRACs to demographic/clinical predictors of CAD}

Further, a statistical covariate analysis was conducted, observing that within the LOW or MID+groups, the TRACs were not significantly affected by the clinical variables. The CAD status was highly significantly related to TRAC score $(p=7.78 \mathrm{E}-11)$, while among the other risk factors for $C A D$, only age was a significant factor for TRAC score $(p=0.012)$, thus confirming that the TRACs appear to be largely independent of known risk factors for CAD in this cohort (Additional file 1: Fig. S2). Exploring the bivariate relationship between age and the TRAC score, there is a slightly negative slope of -1.22 and $R^{2}$ of $0.056(\mathrm{p}=0.021, \mathrm{n}=96$, Additional file 1: Fig. S3). This impact of age is consistent with the use of age and sex in other gene expression models of CAD [22].

\section{Relationship of TRACs to analytical variables}

In addition to the clinical covariates, the potential contribution of analytical/technical variables was considered. Two factors were identified that might affect the types of transcripts: 1) the MID+ patients tended toward lower RNA yield and 2) fewer informative (non-rRNA) reads $(\mathrm{LOW}=8.7 \mathrm{M}$ reads, $\mathrm{MID}+=6.7 \mathrm{M}$ reads, $\mathrm{p}=0.02)$. The likely cause of this difference is the observed difference in lymphocyte counts between groups, which is the primary source of RNA yield (Additional file 1: Fig. S1), and potentially in read depth. To determine whether read depth could contribute to the DEGs, the patients with read depth of $<5 \mathrm{M}$ informative reads $(\mathrm{n}=25)$ were compared to patients with $>5$ M reads $(n=71)$ and analyzed in a similar manner for DEGs. Not surprisingly, a large number of differentially expressed transcripts were identified (1008). However, only 8 transcripts from the 198 TRAC list were sensitive to read depth (APOL4, APTX, C5ORF60, HIF3A, MYO19, NPAS2, RRP12, TMEM67), and this is somewhat confounded by an increased number of MID+ in the low depth group. Thus, it is unlikely that read depth explains the observed pattern of expression in the TRACs.

\section{Interpreting the TRAC signature}

To understand the TRAC signature, the 198-transcript list (Additional file 2: Table S1), generated by analysis of the complete cohort, was subjected to an in-depth

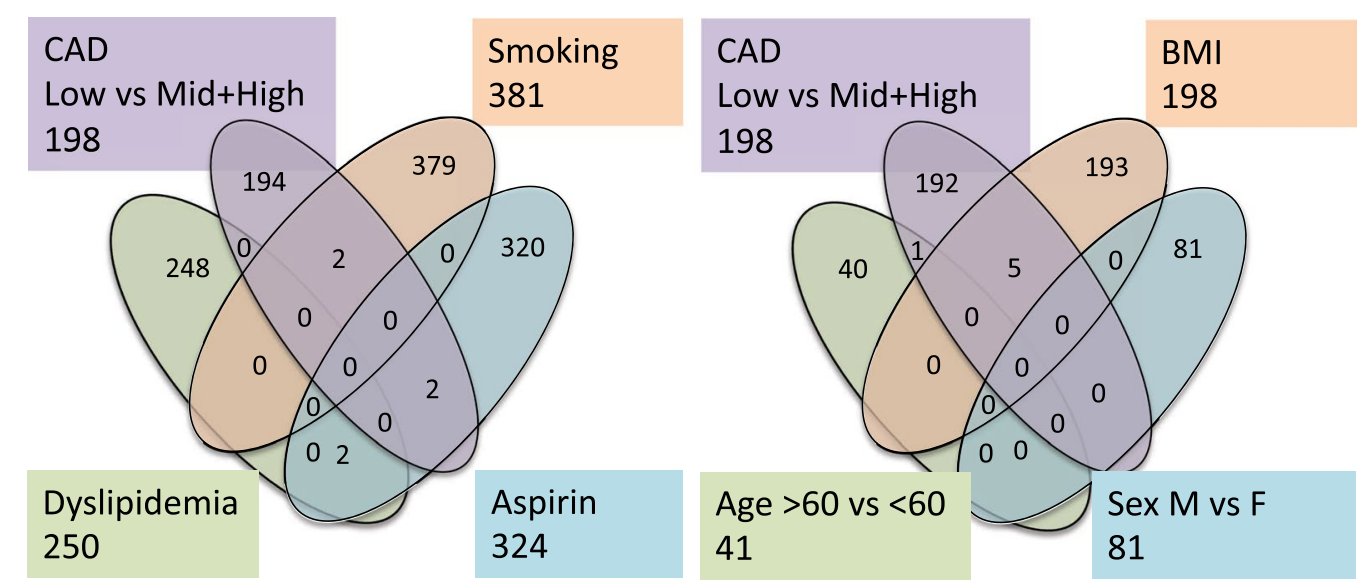

Fig. 3 Relationship between TRACs and transcripts identified for clinical risk factors. To determine whether the TRACs (CAD, LOW vs MID+ High, 198 transcripts) were sensitive to known risk factors for CAD, the 96 patients were separated into new groups based on their current smoking (yielding 381 transcripts), aspirin use (324), dyslipidemia (250), age (41), sex (81), and BMI (198). In the case of age, sex, and BMI (right cluster), only the LOW CAD patients were analyzed $(n=48)$ to prevent confounding with CAD 
analysis. A surprising finding was that 195 of 198 transcripts (98.5\%) were down-regulated in the MID and $\mathrm{HIGH}(\mathrm{MID}+)$ CAD patients, a pattern that rarely occurs in RNA expression analysis, where there is typically a balance between increased and decreased transcripts. Furthermore, the changes are essentially all of the same magnitude (mean $=\sim 1.7$ fold). A similar, but slightly less stringent analysis, using a T-test/fold change filter between the LOW vs MID+ groups identified 461 transcripts, largely overlapping with the 198-gene list, but containing some additional markers of interest, including FLYWCH1, as discussed below (Additional file 2: Table S3).

\section{Discriminant ability of TRACs for clinical CAD}

A partial least squares discriminant (PLSD) model build on these 198 transcripts was very accurate at discrimination between groups, showing an overall accuracy of 98.9\% (100\% for LOW, $97.9 \%$ for MID+). This remained fairly robust even with $\mathrm{N}$-fold internal validation, yielding overall accuracy of $80 \%$ (77\% for LOW, $83 \%$ for MID+). Using a smaller 96 transcript set, with higher fold change, did not improve the predictive ability of the PLSD model built on it, with overall accuracy of 93\% (92\% for LOW, $94 \%$ for MID+), but still produced a quite powerful test, with fewer transcripts. However, these complex polynomial models are able to fit almost any classification, and thus, to minimize 'over-fitting', a much simpler linear model was built using predetermined transcripts connected to $\mathrm{T}$ cell function.

This smaller linear model employed 7 transcripts based on known relevance to $\mathrm{T}$ cell function (DGKA, DLG1, ICOSLG, IKZF4/Eos, SMYD3, TCF3, TRIM28) that were normalized to their average expression level, and then an average composite score was calculated (Fig. 4, Upper Right Panel). The composite score of 7 transcripts was highly significant between groups $\left(\mathrm{p}=6.02 \times 10^{-12}\right)$, and a simple linear prediction model yielded a receiver-operator curve (ROC, via JROCFIT [23]) with a C-statistic of 0.873 , sensitivity of $77.4 \%$, specificity of $83.7 \%$ and overall accuracy of $80.2 \%$, with a positive predictive value (PPV) of $85.4 \%$ and negative predictive value (NPV) of $75.0 \%$ (Fig. 4, Lower Right Panel). By comparison, a purely clinical model using 7 predictors had a C-statistic of only 0.636, with $55.6 \%$ sensitivity, $53.3 \%$ specificity, $54.2 \%$ overall accuracy, 41.7\% PPV, and $66.7 \%$ NPV (Fig. 4, Lower Left Panel). A combined clinical (age) and TRAC model yielded a much stronger C-stat of 0.917 .

\section{Expression changes in relation to GWAS findings}

A variety of GWAS studies have been conducted using various types of atherosclerotic disease or strongly related risk factors, and approximately 150 loci have some reported association with CAD. Several of the TRACs were essentially identical to prior GWAS loci containing variants associated with cardiovascular or immune variables (Table 3). For instance, alpha-1-B glycoprotein (A1BG) associates with hepatocyte growth factor levels in the MESA cohort [24], and with adverse cardiovascular outcomes during antihypertensive therapy [25]. C6ORF10 associates with susceptibility to CAD in Chinese Han [26], Cadherin 13 (CDH13) has SNPs which associate with multiple CAD risks [27, 28], COMM domain-containing 5 (COMMD5) has been identified in rodent models as associated with hypertension [29], the fibrillin 3 locus (FBN3) associates with metabolic syndrome in the Framingham cohort [30], and the $\mathrm{FCH}$ and double SH3 domains 2 (FCHSD2) locus has been associated with systemic lupus erythematosus (SLE) [31], an autoimmune disease frequently complicated by aggressive atherosclerosis. The methylenetetrahydrofolate dehydrogenase 1-like (MTHFD1L) Rs6922269 SNP predicts mortality after acute coronary syndrome [32], and is a known risk loci for CAD [33]. Phospholipase A2 group 10 (PLA2G10) is also a known CAD risk loci in humans [34] and mice [35]. Psoriasis is a well-established risk for $\mathrm{CAD}$, and the psoriasis susceptibility 1 candidate 1 (PSORS1C1) gene expression is reduced in the present CAD cohort, and its locus is associated with psoriasis [36], rheumatoid arthritis [37], and capillary leak [38], and was recently associated with cardiometabolic parameters [39]. Serpin peptidase inhibitor D (SERPIND1, heparin cofactor II) levels have been associated with in-stent restenosis of peripheral arteries [40], and the staining for SERPIND1 in human coronary lesions was correlated with the degree of atherosclerosis in the PDAY study [41]. Notably, Flywich-type zinc finger 1 (FLYWCH1), was identified by the CARDIOGRAM consortium as a driver eQTL risk loci for CAD in vascular and adipose tissues [42]. Thyroid adenoma associated (THADA) was identified in a functional expression analysis of a human beta cell line as potentially relevant to type II diabetes [43]. Thus, GWAS and expression studies suggest that several of the whole blood mRNA expression changes correspond with previously published SNPs for CAD, or CAD risk factors, such as hypertension, SLE, type 2 diabetes, and psoriasis.

\section{Ontology/pathway analysis of TRACs}

The 198-gene list was more fully annotated by both automated and manual literature mining and genome analysis. Several levels of analysis were employed. 

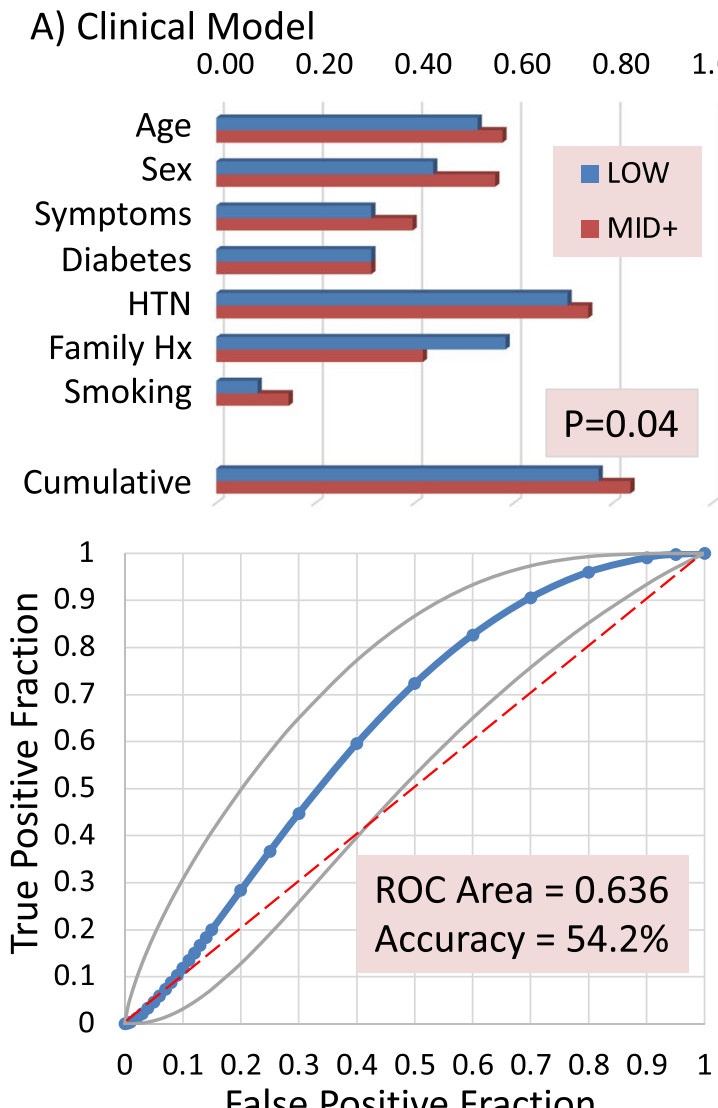

B) TRAC Model

$$
0.00
$$

0.50

1.00

1.50
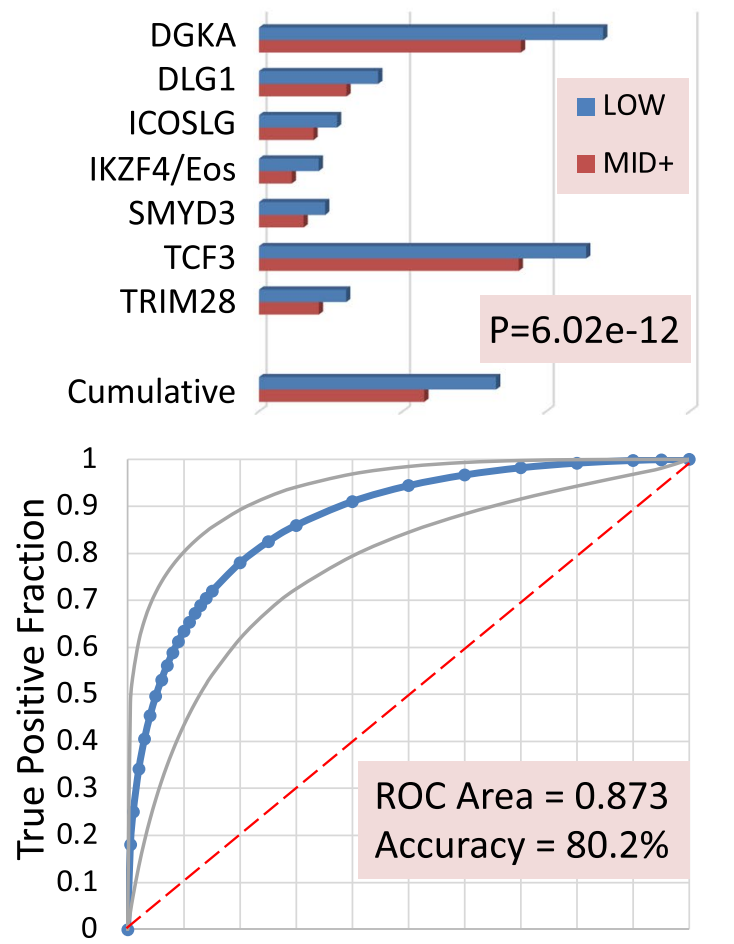

$\begin{array}{lllllllllll}0.0 & 0.1 & 0.2 & 0.3 & 0.4 & 0.5 & 0.6 & 0.7 & 0.8 & 0.9 & 1.0\end{array}$ False Positive Fraction

Fig. 4 Clinical versus RNA predictors of CAD. a Conventional clinical predictors of CAD plotted for each group in the upper panel, showing Age (decades/10), Sex (\%Male), Symptom type (typical/atypical), Diabetes (\%), Hypertension (HTN, \%), Family History of CAD (\%), and current Smoking (\%). A cumulative CAD risk score is calculated for each patient based on the method of Min et al. and divided by 10 for graphic purposes. The relationship between the cumulative risk score and CAD was calculated by the Receiver Operator Characteristic (ROC) and a confusion matrix to identify the accuracy of the method (lower left). $\mathbf{b}$ The performance of 7 RNA transcripts as their gene symbols (i.e. DGKA, DLG1) expressed as the RPKM by CAD group. A cumulative score was computed expressing each transcript as a ratio to the mean of its expression in the entire group, to prevent highly expressed transcripts from being over-represented. For graphic purposes, the TRIM28 and Cumulative scores are /10. In the lower panel, the relationship between the cumulative TRAC score (constant-TRAC, to create positive ROC) and angiographically-confirmed CAD is analyzed by ROC similar to the clinical model for the 48 patients in each group

Initially, because the DEGs tended to all be decreased by a similar magnitude, transcripts were examined to determine whether they were indicative of a particular cell type present in blood that might be associated with CAD. At least 17 of the TRACs were readily associated with T-cell function (Table 4, upper). Notably, CYTIP and PLCG1 have known interactions with the $\mathrm{T}$ cell receptor (TCR) signaling $[44,45]$. Likewise, DLG1 and PPARA are well established regulators of T-cell function, and TIA1 is an intracellular antigen which marks cytotoxic T-cells [46-48].

As shown in the lower half of Table 4, another 10 transcripts suggest that TRACs might be most closely associated with regulatory $\mathrm{T}$ cells (Treg). Several strong indications are provided by transcripts such as IKAROS family zinc finger 4 (IKZF4, aka Eos), which is considered a signature transcript for the Treg cell subset [49], and which is important in controlling Treg transition into T-helper (Th) cells [50]. IKZF4/Eos is thought to be a required corepressor for the FoxP3-dependent gene silencing that is necessary for maintaining the stable Treg phenotype [51]. Likewise, Set and Mind domain containing 3 (SMYD3) is also involved in epigenetic control of FoxP3 expression [52]. Further, TCF3, aka E2A, is a major transcription factor controlling FoxP3 expression [53], and TRIM28 has been identified as a member of the FoxP3 transcriptional complex [54]. Given that FoxP3 is considered the hallmark of Treg cells [55], alterations in the expression of 
Table 3 TRACs with known GWAS or expression associations

\begin{tabular}{|c|c|c|c|c|c|c|c|c|c|}
\hline \multirow[t]{2}{*}{ Gene name } & \multirow[t]{2}{*}{$p$ value } & \multirow{2}{*}{$\begin{array}{l}\text { Fold } \\
\text { HvsL }\end{array}$} & \multirow{2}{*}{$\begin{array}{l}\text { Fold } \\
\text { MvsL }\end{array}$} & \multicolumn{3}{|c|}{ Expression (RPKM) } & \multirow[t]{2}{*}{ Symbol } & \multirow[t]{2}{*}{ Description } & \multirow[t]{2}{*}{ RefSeq } \\
\hline & & & & $\mathrm{HIGH}$ & MID & LOW & & & \\
\hline uc061drv.1 & 2.93E-04 & $1.01 \uparrow$ & $-4.97 \downarrow$ & -1.24 & -3.56 & -1.25 & $\mathrm{~A} 1 \mathrm{BG}$ & alpha-1-B glycoprotein & NM_130786 \\
\hline uc059ulu.1 & 5.07E-04 & $-1.57 \downarrow$ & $-2.22 \downarrow$ & -0.71 & -1.21 & -0.06 & BBS2 & Bardet-Biedl syndrome 2 & NM_031885 \\
\hline uc061wwa.1 & $6.36 \mathrm{E}-04$ & $-1.44 \downarrow$ & $-1.39 \downarrow$ & -0.29 & -0.23 & 0.24 & BLCAP & Bladder cancer associated protein & NM_001167820 \\
\hline uc063nqr.1 & $3.79 E-04$ & $1.29 \uparrow$ & $-3.35 \downarrow$ & -5.67 & -7.78 & -6.04 & C6orf10 & Chromosome 6 open reading frame 10 & NM_001286474 \\
\hline uc059xrj.1 & $1.45 \mathrm{E}-04$ & $-2.97 \downarrow$ & $-8.68 \downarrow$ & -2.86 & -4.41 & -1.29 & $\mathrm{CDH} 13$ & Cadherin 13 transcript variant 5 & NM_001220491 \\
\hline uc064rqz.1 & $8.12 \mathrm{E}-04$ & $-1.46 \downarrow$ & $-1.57 \downarrow$ & 0.09 & -0.01 & 0.64 & COMMD5 & COMM domain containing 5 & NM_001081004 \\
\hline uc060std.1 & $7.00 \mathrm{E}-04$ & $1.21 \uparrow$ & $-6.37 \downarrow$ & -2.71 & -5.66 & -2.99 & FBN3 & fibrillin 3 & NM_032447 \\
\hline uc058fep.1 & $6.63 \mathrm{E}-04$ & $-1.37 \downarrow$ & $-1.52 \downarrow$ & -0.02 & -0.18 & 0.43 & FCHSD2 & $\mathrm{FCH}$ and double $\mathrm{SH} 3$ domains 2 & NM_014824 \\
\hline uc058vgw.1 & 2.07E-04 & $-1.69 \downarrow$ & $-1.83 \downarrow$ & -2.04 & -2.15 & -1.28 & MMP17 & Matrix metallopeptidase 17 & NM_016155 \\
\hline uc063sgp.1 & $7.99 \mathrm{E}-04$ & $-2.35 \downarrow$ & $-6.59 \downarrow$ & -2.57 & -4.06 & -1.34 & MTHFD1L & Methylenetetrahydrofolate dehydrogenase $1 \mathrm{~L}$ & NM_001242768 \\
\hline uc059rbn.1 & $8.11 \mathrm{E}-04$ & $-1.42 \downarrow$ & $-1.91 \downarrow$ & 1.87 & 1.45 & 2.38 & PLA2G10 & Phospholipase A2, group X & NM_003561 \\
\hline uc063mxz.1 & $3.53 \mathrm{E}-04$ & $-4.06 \downarrow$ & $-5.22 \downarrow$ & -3.46 & -3.82 & -1.44 & PSORS1C1 & Psoriasis susceptibility 1 candidate 1 & NM_014068 \\
\hline uc059xen.1 & $9.57 \mathrm{E}-04$ & $-1.43 \downarrow$ & $-1.59 \downarrow$ & -0.58 & -0.73 & -0.06 & RFWD3 & Ring finger and WD repeat domain 3 & NM_018124 \\
\hline uc062bvk.1 & $8.81 \mathrm{E}-04$ & $-1.52 \downarrow$ & $-1.42 \downarrow$ & -0.80 & -0.69 & -0.19 & SERPIND1 & Serpin peptidase inhibitor $D$, heparin cofactor & NM_000185 \\
\hline uc001zwr.5 & $9.10 \mathrm{E}-04$ & $1.02 \uparrow$ & $1.67 \uparrow$ & 0.59 & 1.30 & 0.56 & SLC12A1 & Solute carrier family $12 \mathrm{~A} 1$ & NM_000338 \\
\hline uc064wao.1 & $3.99 \mathrm{E}-04$ & $-1.80 \downarrow$ & $-1.66 \downarrow$ & -1.08 & -0.96 & -0.23 & SLC25A25 & Solute carrier family 25 A25 & NM_052901 \\
\hline uc063fxo.1 & $5.55 \mathrm{E}-04$ & $1.02 \uparrow$ & $-1.81 \downarrow$ & 0.47 & -0.41 & 0.44 & SLC25A46 & Solute carrier family 25 member 46 & NM_001303250 \\
\hline uc062iym.1 & $2.16 \mathrm{E}-04$ & $-2.20 \downarrow$ & $-1.14 \downarrow$ & -0.19 & 0.76 & 0.95 & SLC6A20 & Solute carrier family 6 (proline transport) & NM_022405 \\
\hline uc061irb.1 & $5.78 \mathrm{E}-05$ & $-2.01 \downarrow$ & $-1.74 \downarrow$ & -1.11 & -0.91 & -0.10 & THADA & Thyroid adenoma associated & NR_073394 \\
\hline uc060vxm.1 & $4.76 \mathrm{E}-06$ & $-3.08 \downarrow$ & $-10.76 \downarrow$ & -2.48 & -4.28 & -0.86 & TMEM161A & TRANSMEMBRANE protein 161A & NM_001256766 \\
\hline uc061you.1 & 1.46E-04 & $-1.45 \downarrow$ & $-1.61 \downarrow$ & -0.91 & -1.07 & -0.38 & ZGPAT & Zinc finger, $\mathrm{CCCH}$-type with G patch domain & NM_181485 \\
\hline
\end{tabular}

these transcripts suggest that changes in the abundance of the Treg population may contribute to the TRAC signature.

\section{Cell type-specific RNA markers in relation to CAD level}

To explore a potential cell type hypothesis more directly, published microarray analysis of purified human blood subsets have identified cell type-specific mRNAs [56], which were cross-referenced to the current RNAseq transcriptome, and used to build a composite index of $\sim 15$ to 20 mRNAs relatively unique to each subtype. As shown in Fig. 5, a composite index of RNA expression levels shows a trend toward lower expression of lymphocyte markers in patients with MID to HIGH CAD. This trend is maintained in T-cells, and specifically in CD8 + T-cells, but is not observed in B-cell or granulocyte-related transcripts.

\section{TRACs do not appear to be markers of circulating} progenitor cells

There is a substantial literature [57], summarized in Additional file 2: Table S4, that consistently reports reductions in circulating progenitor cell (CPC) populations in patients with stable CAD $[58,59]$, or preclinical atherosclerosis [60]. The major cardiovascular risk factors are associated with reduced numbers and activity of CPC
[61]. Conversely, circulating endothelial progenitor cells (EPC) are increased in acute MI cases [62]. However, it is unlikely that a decrease in EPC numbers, which are rare $(<1 \%)$, could cause the substantial shift in RNA levels, detected in whole blood. Nonetheless, the RNAseq data was analyzed for changes in recognized markers of EPC and CPC, such as CD34, cKit, PROM1/AC133, and KDR, and the RNA levels are shown in Additional file 1: Fig. S4. There was no systematic change detectable: CD34 and cKit were slightly elevated in MID + CAD, while KDR and $\mathrm{AC} 133$ were decreased by comparable amounts.

\section{The expression of consensus Treg markers by CAD level}

A second potential explanation for TRACs as markers of a specific cell type is that there are known reductions in the Treg subset of lymphocytes in atherosclerosis [63]. An extensive literature documents reduced Treg abundance, and a relative imbalance in Treg vs $\mathrm{T}$ effector (Teff) cells in patients with CAD (summarized in Additional file 2: Table S5) $[63,64]$. To test for the potential changes in Treg, the mRNA levels of known Treg markers was analyzed in the CAD groups. As shown in Fig. 6, five established markers of Treg cells, FoxP3, CD4, CD25, ETS1, and RUNX1, showed a stepwise decrease in mRNA expression from LOW, MID, to HIGH CAD. By 
Table 4 TRACs related to T cell and Treg function

\begin{tabular}{|c|c|c|c|c|c|}
\hline Gene name & p value & Fold HvsL & Symbol & Description & RefSeq \\
\hline \multicolumn{6}{|c|}{ Related to T cell function } \\
\hline uc064mjf.1 & $8.03 \mathrm{E}-04$ & $-1.24 \downarrow$ & AP3M2 & adaptor-related Protein 3, mu 2 & NM_006803 \\
\hline uc061mig.1 & $6.28 \mathrm{E}-04$ & $-1.21 \downarrow$ & CHST10 & Carbohydrate sulfotransferase 10 & NM_004854 \\
\hline uc061otq.1 & 1.03E-04 & $-1.47 \downarrow$ & CYTIP & Cytohesin 1 interacting protein & NM_004288 \\
\hline uc058pdy.1 & 7.75E-04 & $-1.36 \downarrow$ & DGKA & Diacylglycerol kinase, alpha & NM_201554 \\
\hline uc062seb.1 & $9.10 \mathrm{E}-04$ & $-1.18 \downarrow$ & DLG1 & DISCS, large homolog 1 & NM_001204386 \\
\hline uc057gll.1 & $5.73 \mathrm{E}-04$ & $-1.73 \downarrow$ & EPS15 & EGF receptor pathway substrate 15 & NM_001981 \\
\hline uc063okh.1 & $1.65 E-04$ & $1.12 \uparrow$ & FOXP4-AS1 & FOXP4 antisense RNA 1 & NR_126417 \\
\hline uc062dbe.1 & $3.38 \mathrm{E}-04$ & $-1.56 \downarrow$ & GATSL3 & GATS protein-like 3 & NM_001037666 \\
\hline uc059uvj.1 & $5.04 \mathrm{E}-04$ & $1.44 \uparrow$ & GPR56 & Adhesion G prot-coupled recep G1 & NM_001145774 \\
\hline uc062xlo.1 & $2.87 \mathrm{E}-04$ & $-1.15 \downarrow$ & NUP54 & Nucleoporin 54 kDa & NR_103781 \\
\hline uc057jvt.1 & $6.40 \mathrm{E}-04$ & $-1.51 \downarrow$ & PHGDH & Phosphoglycerate dehydrogenase & NM_006623 \\
\hline uc061xai.1 & 5.74E-04 & $-1.75 \downarrow$ & PLCG1 & Phospholipase C, gamma 1 & NM_182811 \\
\hline uc062fel.1 & $1.70 \mathrm{E}-04$ & $-1.09 \downarrow$ & PPARA & perox. prolif. activated rec. a & NM_001001928 \\
\hline uc058jqr.1 & $9.54 \mathrm{E}-04$ & $-1.26 \downarrow$ & RAD52 & RAD52 homolog & NM_001297421 \\
\hline uc064bpk.1 & $5.85 \mathrm{E}-04$ & $-1.30 \downarrow$ & SCIN & Scinderin & NM_033128 \\
\hline uc061kij.1 & $8.52 \mathrm{E}-04$ & $-1.45 \downarrow$ & $\mathrm{TIA} 1$ & Cytotoxic granule-assoc. RNA BP & NM_022173 \\
\hline \multicolumn{6}{|c|}{ Relevant to Treg and/or FoxP3 } \\
\hline uc063bvz.1 & $9.62 \mathrm{E}-05$ & $-1.25 \downarrow$ & AHRR & Aryl-hydrocarbon rec. repressor & NM_001242412 \\
\hline uc058uor.1 & $8.95 \mathrm{E}-04$ & $-1.38 \downarrow$ & HIP1R & Huntingtin interact. prot.1 related & NM_003959 \\
\hline uc061yty.1 & $1.48 \mathrm{E}-04$ & $-1.62 \downarrow$ & ICOSLG & Inducible T-cell costimulator ligand & NM_001283052 \\
\hline uc058pgk.1 & $8.59 \mathrm{E}-04$ & $-3.36 \downarrow$ & IKZF4 & IKAROS family zinc finger 4 (Eos) & NM_022465 \\
\hline uc063ljh.1 & $9.84 \mathrm{E}-04$ & $-1.59 \downarrow$ & IRF4 & Interferon regulatory factor 4 & NM_001195286 \\
\hline uc063ady.1 & $5.93 \mathrm{E}-04$ & $-1.34 \downarrow$ & LRBA & LPS-responsive, beach anchor & NM_006726 \\
\hline uc057qye.1 & 2.67E-04 & $-1.35 \downarrow$ & SMYD3 & SET and MYND domain containing 3 & NM_022743 \\
\hline uc057jсу.1 & $5.62 \mathrm{E}-04$ & $-1.55 \downarrow$ & STRIP1 & Striatin interacting protein 1 & NM_001270768 \\
\hline uc061duf.1 & $4.36 \mathrm{E}-04$ & $-1.33 \downarrow$ & TRIM28 & Tripartite motif containing 28 & NM_005762 \\
\hline uc060rek.1 & 4.39E-04 & $-1.39 \downarrow$ & TCF3 & Transcription factor 3 & NM_003200 \\
\hline
\end{tabular}

comparison, the expression of an irrelevant marker, such as the prostaglandin E receptor 3 (PTGER3), does not show this CAD-related trend.

\section{TRACs correlate with FoxP3 and other Treg markers}

To further understand whether the TRACs are related to Treg cell changes, the expression levels of FoxP3 were correlated with 24 other known Treg markers or TRACs across all 96 patients (Additional file 2: Table S6). The strongest correlations of FoxP3 occurred with AHRR (0.72, TRAC), CD8A (0.53), PDCD1 (0.48), ICOSLG (0.41, TRAC), RUNX1 (0.36), and PSORS1C1 (0.35, TRAC) (all $\mathrm{p}<0.001$ ), while other known Treg markers, such as IKZF4/Eos (0.13, p >0.2, TRAC), showed weaker correlations. For reference, 2 splice variants of ICOS are correlated at 0.87 , and 2 variants of B3GAT1 are correlated at 0.64. Furthermore, the levels of ICOS and ICOSLG mRNA in whole blood are reduced comparably to FoxP3 in both the MID and HIGH CAD groups (Additional file 1: Fig. S5). Thus, the expression of several of the TRACs (AHRR, ICOSLG, PSORS1C1), correlate with FoxP3 RNA levels in these patients to a degree similar to or better than other known Treg markers (RUNX1, IKZF4/Eos).

\section{Treg/Teff cell ratio relative to TRAC RNA expression}

To determine whether a reduction in Treg cell counts in blood would be sufficient in magnitude to produce the observed changes in RNA levels, 8 publications that reported Treg percentages in normal and CAD patients, such as unstable angina or acute coronary syndrome (ACS), were reviewed, and the change in Treg percentage was computed (Additional file 2: Table S5). The average Treg abundance, typically defined as $\mathrm{CD} 4{ }^{+} \mathrm{CD} 25^{+} \mathrm{CD} 127^{\text {low }}$ by flow cytometry, was $4.7 \%$ in normal, but decreased to $3.2 \%$ in CAD or unstable angina (30.3\% reduction). This reduction in Treg abundance would translate to a 1.43-fold difference in Treg RNA levels, assuming that these markers are relatively unique to Tregs. Thus, the 1.47-fold reduction in mRNA for the 


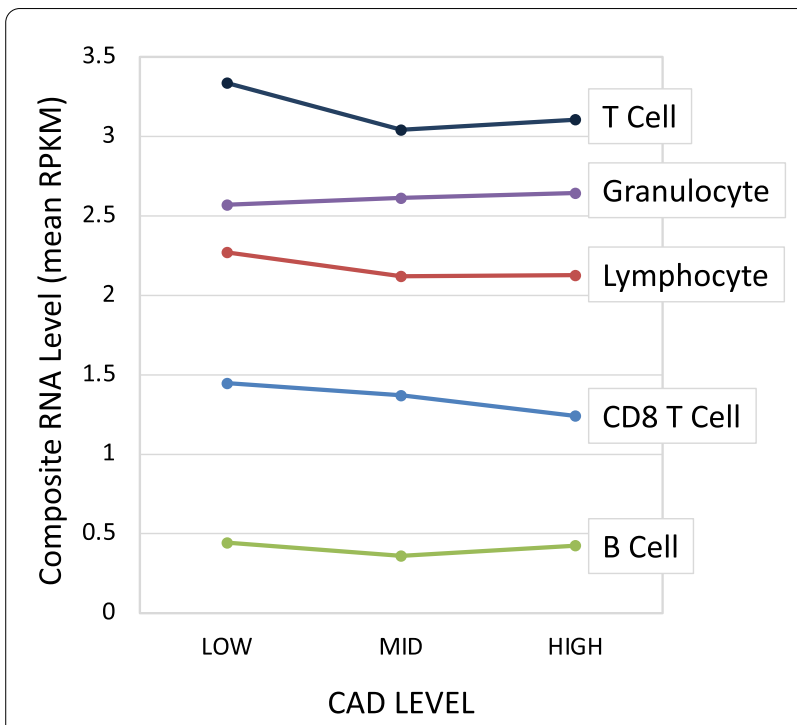

Fig. 5 Expression of cell-type specific transcripts as a function of CAD status. Transcripts with relative specificity toward particular blood cell subsets was curated from published studies. The expression level (RPKM) of those transcripts (10-15 per cell type) in the RNAseq data was calculated and averaged for each cell type. The average expression was calculated for patients in 3 groups of CAD severity, $\operatorname{LOW}(n=48), \operatorname{MID}(n=28)$, or HIGH $(n=20)$ consensus FoxP3 marker, and the $~ 1.7$-fold reduction in the TRACs, is quite consistent with the reported reduction in Treg cell numbers in CAD.

\section{TRACs and Treg markers are sensitive to RNA stabilization procedures}

Given that the Treg imbalance data has been reported from multiple labs worldwide, it is curious that changes in established Treg markers have not been reported in any prior publications using expression profiling of blood from patients with stable CAD. One possible explanation is that RNAseq is potentially much more sensitive than microarray methods, allowing these low abundance messages to be detected more accurately. A second consideration is that, to our knowledge, all prior CAD microarray studies were conducted using RNA stabilized and isolated from Paxgene preservative tubes, while the present studies employed Tempus preservative tubes. In the current studies, Tempus tubes were selected due to studies in our lab, and others, showing a 10 to $20 \%$ better yield of RNA at $20 \%$ lower cost and $40 \%$ less time [65, 66]. Based on prior studies demonstrating quite marked changes in gene expression profiles based on the RNA stabilizer [20, $65,66]$, the effect of the RNA stabilizer was examined for its impact on TRACs versus neutrophil transcripts (DEFA3) and other selected markers (IL12A, SELL,
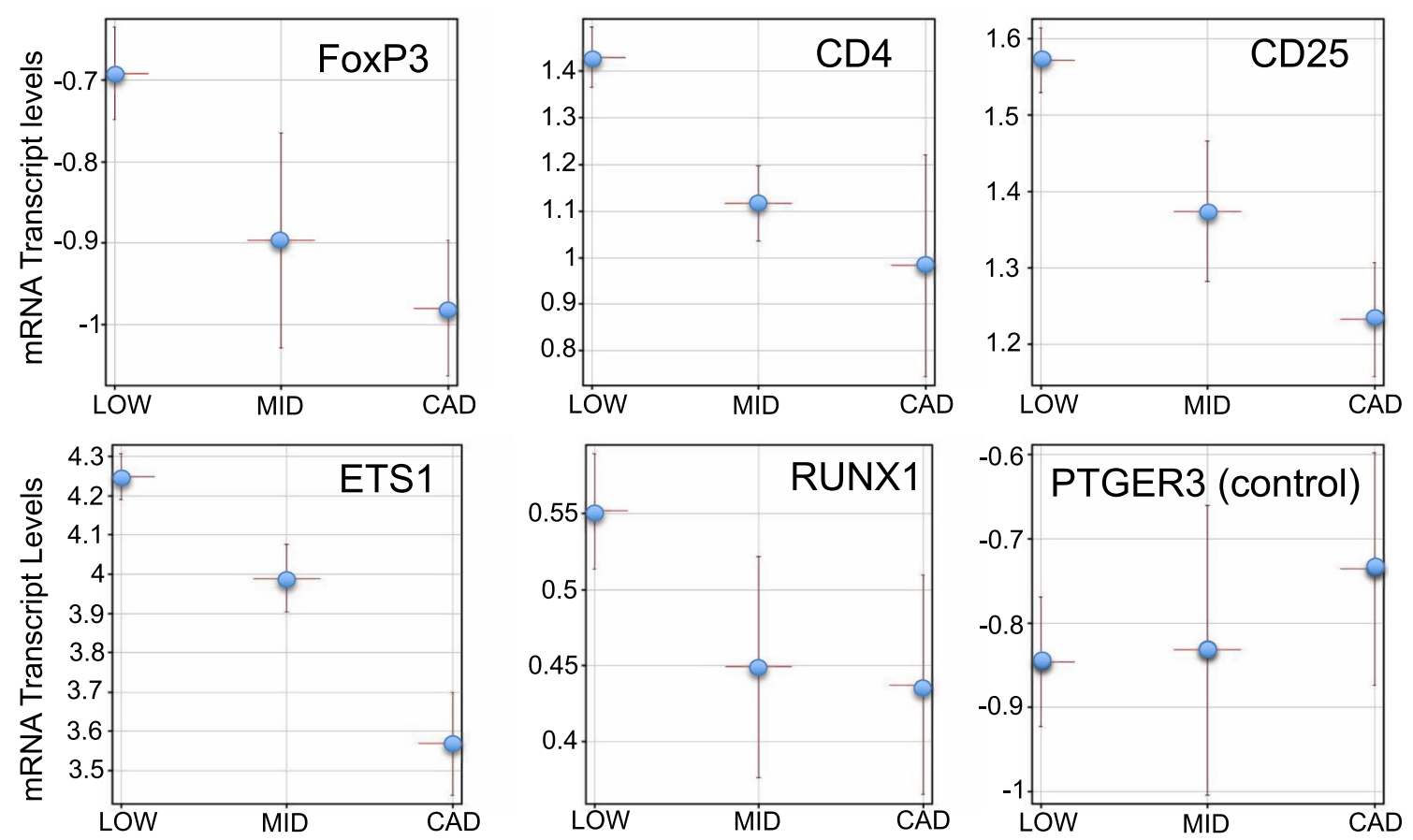

Fig. 6 RNA levels of markers for Treg cells as a function of CAD level. The expression levels ( $\log _{2}$ RPKM) of 5 known Treg markers (FoxP3, CD4, CD25, ETS1, Runx1) and 1 control (PTGER3) is plotted for 3 groups of patients with LOW ( $\leq 20 \%$ stenosis, $n=48)$, MID (21-69\% stenosis, $n=28)$, or high $\mathrm{CAD}(\geq 70 \%$ stenosis, $n=20)$. Points are mean per group with bars \pm s.e.m 
SOD2, TBA1, ACTB). As shown in Additional file 1: Fig. S6, blood from the same normal subjects at the same time, but collected into two different collection/stabilizer tubes, showed marked differences in the levels of mRNAs measured by droplet digital PCR (ddPCR). Several of the TRACs, such DGKA, DLG1, ICOSLG, and TCF3, were detected $\sim 4$ to sixfold more efficiently in blood RNA isolated from Tempus versus Paxgene tubes. Chemically, the Paxgene tubes are based on a cationic detergent that creates micellar-like structures that protect RNA, while Tempus uses the strong chaotrophic effects of guanidinebased salts to denature RNAses and dissolve RNA/protein complexes. Thus, the Tempus/chaotrophic approach appears to isolate Treg-related mRNAs better than the Paxgene/detergent approach.

\section{Analysis of TRACs in an independent validation cohort}

Simple linear classification models built in the Discovery cohort (Fig. 4) and then applied to the RNAseq values obtained from 80 patients in the Validation Cohort did not perform much better than random in the validation set. However, it was quickly noted that the Discovery RNAseq had been aligned to the GRCh37/hg19 human genome, while the Validation set, aligned at a later date, used the GRCh38/hg38 reference genome. Thus, the entire Discovery RNAseq database was realigned to the hg38 genome and then reanalyzed for DEGs to build a classification model. As a quick test of the stability of a predictive model, in the hg38-aligned Discovery dataset, strict filtering for $>$ twofold change at $\mathrm{p}<0.001$ identified 27 transcripts of which $23(85 \%)$ were expressed at a lower level in the CAD group. A PLSD model built on those 27 transcripts was $95.5 \%$ accurate in LOW, 91.9\% accurate in MID+, for $93.3 \%$ overall accuracy. However, those same transcripts were less predictive in the Validation dataset, but still informative, showing $78.4 \%$ accuracy for low, $62.8 \%$ for MID+, with $70 \%$ overall accuracy. Thus, the hg19 vs hg38 alignments play a significant role in the stability of the TRAC signal, but the discriminant ability of PLSD models remains imperfect between cohorts. To understand this discrepancy, the DEGs identified by each cohort were analyzed.

\section{Correlation between Discovery and Validation expression levels for TRACs}

Using the list of 599 DEG transcripts identified in the Validation set, it was determined that their expression levels in the Discovery set were highly correlated for both the low $(r=0.96)$, and Mid $+(r=0.98)$ CAD groups. Thus, quantitation of the transcript levels in the 2 cohorts was very similar, at least at the group level (LOW vs MID+). Thus, the variation in the DEGs between the 2 cohorts is more likely attributable to variation at the patient to patient level, which could reflect the different demographics of the 2 groups.

\section{Identification of TRACs shared by the discovery and validation cohorts}

Using the strictest filtering, exactly as applied to the Discovery set to obtain the $27 \mathrm{~g}$ predictors used above, the Validation dataset yielded 22 transcripts, but none were identical matches at the gene symbol level between cohorts. By relaxing the filtering criteria to create DEGs of about 350 unique and annotated transcripts in each cohort $(p<0.01, f c 1.2), 16$ exact matches were observed, which is 4.5 times greater than expected by chance $\left(\mathrm{p}<8.7 \times 10^{-7}\right)$ (Additional file 2: Table S7). An additional 17 close matches were observed (ie. ELP3 vs ELP2), and 37 more matches that were close or identical to HG19 alignments of the Discovery cohort, for a total of 70 close or exact matches. Both the Discovery and Validation DEGs (92\% decreased in CAD) shared a strong trend toward decreasing expression in the CAD group.

\section{Cell type analysis in reproducible TRACs}

These transcripts common to both datasets were used to determine if any enrichment of a particular cell type was evident by comparing them to the precurated Blood Atlas RNAseq database. The results indicated the greatest similarity to $\mathrm{T}$ cells, with 12 exact or close matches (4.3-fold over-representation, $\mathrm{p}=9.8 \times 10^{-6}$, Fisher Exact test). Rather striking in this group of $\mathrm{T}$ cell-related transcripts, identified as significantly decreased in both cohorts, is FoxP1. While FoxP3 is considered a pivotal transcript in Treg development, FoxP1 is likewise a well-known and critical determinant of Treg maturation [67].

By comparison, the overlap of the shared DEG list with other cell types is less striking: B cell (1 exact, 4 close matches, 3.5 fold enrichment, $\mathrm{p}<0.014$ ), granulocytes ( 1 exact, 5 close matches), monocyte/macrophage (1 exact, 4 close matches), natural killer (NK) cells (1 close match), dendritic cells (3 exact, 4 close matches). Some transcripts, especially OSBPL10, were found as an exact match on multiple cell types, and thus do not truly inform the cell type analysis.

\section{Prevalence of transcripts associated with stress granules} (SG)

In addition to the apparent similarity of TRACs with Treg markers, it was also noted that a disproportionate number of transcripts had a known association with stress granules (Additional file 2: Table S8). Stress granules are membrane-less granules that result from liquid to gel transitions under cellular stress, and contain RNAs that are being sequestered from translation during various stressors, such as nutrient stress. Fortunately, other 
groups have used relatively unbiased approaches, such as microarrays and RNAseq, to identify RNA transcripts retained in SG during stress [68]. Thus, this hypothesis was tested more formally by comparing the TRACs to known SG transcripts and determining whether the overlap was greater than expected by chance.

In the initial hg19 TRAC list (198 transcripts, Additional file 2: Table S1) there was noticeable similarity to previously published lists of SG transcripts [68]. For instance, of the 198 TRACs, 34 were near or exact matches to known SG transcripts, reflecting a fivefold overrepresentation $\left(\mathrm{p}=9.5 \times 10^{-15}\right)$. This association held strong when the TRACs common to both studies were analyzed for their similarity to a previously curated list of 723 known SG transcripts, whereby there was a 25 -fold enrichment for SG transcripts $\left(\mathrm{p}=4.04 \times 10^{-39}\right)$. A summary of these transcripts is shown in Additional file 2: Table S8, and 10 transcripts are depicted graphically in Fig. 7.

The stress granule-related RNAs include dead box proteins (DDXs, including DDX46, DDX51, DDX54), which are a family of RNA helicases that regulate RNA biogenesis, editing, folding, translation, and decay, as well as having critical antiviral activities [69]. Likewise, EDC3 is considered an important regulator of mRNA translation and decay [70], and interestingly, DDX proteins

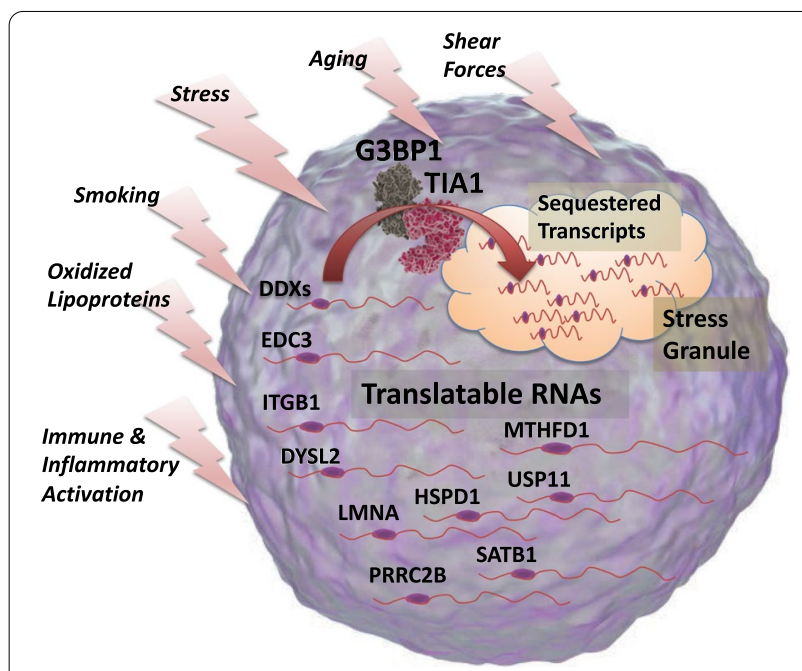

Fig. 7 Schematic representation of stress granule-regulated transcripts. Analysis of the transcripts associated with CAD (TRACs) indicated an apparent enrichment for transcripts previously known to be associated with stress granules, which are membrane-less aggregates of proteins and RNA formed when cells are exposed to a variety of stressors, listed on the left. Under stress, these TRACs, of which 10 are shown here (DDX, EDC3, etc.), translocate from active, translatable forms in the cytosolic machinery, to sequestered, inactive forms in the stress granule. Molecular images courtesy of www.somer sault1824.com under a Creative Commons license (i.e. DDX6) are known partners to EDC3 and mRNA decapping enzymes in the regulation of P-body assembly and function [71]. Of note is the Lamin A (LMNA) transcript, which is the target of germline mosaic mutations in Hutchinson-Guilford Progeria, a premature aging syndrome characterized by aggressive atherosclerosis and myocardial infarction in adolescents [72]. Also of interest, special AT-rich binding protein (SATB1) is a key chromatin protein that is a well-established modulator of $\mathrm{T}$ cell progenitor maturation [73]. Notably, SATB1, along with IKZF4/Eos, IRF4, and GATA1, are considered a Treg 'locking' genes [74].

\section{Potential involvement of cilia/immune synapse transcripts}

During manual curation of the DEG transcripts from both cohorts, there was an apparent overrepresentation of transcripts related to cilia, synapses, and adhesion: functions not normally associated with circulating cells. A representative list of 11 such transcripts derived from the DEGs common to both cohorts is shown in Additional file 2: Table S9. An excellent example is BardetBiedl Syndrome 2 (BBS2) which is a heritable cause of an autosomal recessive syndrome characterized by central obesity, rod-cone dystrophy, renal and vascular abnormalities that emanate from a central defect in cilia assembly and synaptic function [75]. Related transcripts that appeared in only one of the cohorts includes dystonin (DST), which likewise affects the ciliary connections in the ear, causing congenital deafness, but has also been associated by GWAS with CAD [76]. Other DEGs common to both cohorts include copine 3 or 6 (CPNE3/6) which are components of the ciliary body, and affects neural plasticity, but coincidentally, reduced CPNE3 expression is associated with the risk of acute MI and stable CAD [77]. A potential connection between these cilia/synaptic transcripts and the Treg changes in atherosclerosis is that the maturation of Tregs likely depends on proper immune synapse formation in maturing $\mathrm{T}$ cells [78].

\section{Comparison of TRACs to prior microarray-based biomarker panels}

Other published works have identified transcripts with predictive value for CAD based on Affymetrix array technology and PaxGene blood RNA preservation tubes [12]. For comparison purposes, these published transcripts were matched by gene symbol to RNAseq transcripts, identifying 17 transcripts in the current RNAseq dataset. The expression levels of these array-based markers were overall much higher than TRACs, but surprisingly, the RNA levels did not differ between LOW and $\mathrm{MID}+$ (average $\log _{2} \mathrm{RPKM}=3.26$ vs $3.31, \mathrm{p}=0.94$ ) (Additional file 2: Table S10). These 17 transcripts were 
used to build a classification model that yielded only $36.7 \%$ accuracy (LOW 45.1\%, MID 10\%, CAD 37.5\%, w/ $33 \%=$ random). However, in fairness to the prior CAD biomarkers, it is difficult to extrapolate their weighting algorithm to the RNAseq data, and that might improve the prediction model.

\section{Discussion}

The analysis of the RNA transcriptome in relation to angiographically confirmed CAD offers several major advantages in both our basic science understanding of $\mathrm{CAD}$ and in clinical medicine. First, if blood biomarkers can be identified, it might be possible to reduce invasive testing, such as cardiac catheterization, as well as more judiciously use imaging resources, such as CT and MR angiography. Secondly, it would be possible to improve diagnosis of CAD in rural areas worldwide, where invasive or advanced imaging methods are unavailable. Finally, the proposed biomarkers potentially can serve both as therapeutic targets and markers to monitor the appropriateness and efficacy of new or existing therapies, such as statins or PCSK9 inhibitors. For instance, Treg numbers have been shown to be responsive to statin therapy, and so it might be possible to use TRACs to monitor statin therapies.

The connection between the immune system and atherosclerosis is extensively documented. Blood components, especially monocytes/macrophages [79], neutrophils, lymphocytes [80], and platelets mechanistically contribute to the development of CAD [81]. Recently, the microanatomy of the human carotid atherosclerotic lesion has been analyzed by single-cell transcriptomics, revealing at least 14 subtypes of cells, including several $\mathrm{T}$ cell subsets [82]. The present results are consistent with the extensive evidence that CAD is associated with changes in the Treg/Teff ratio, lipid imbalances, inflammation, microbiome changes, and autoimmunity in atherosclerosis [83]. There is a large and fairly consistent literature demonstrating changes in the Treg/Teff ratio in patients with CAD [84-88], and the observed cellular changes would be consistent in both direction and magnitude with the detected changes in mRNA expression in the present studies (Additional file 2: Table S5). One interpretation of the beneficial effects of statins is that in addition to lowering LDL cholesterol, statins can induce FoxP3 + Treg cells, via modulation of TGF- $B$ signaling $[89,90]$. Beyond the reproducible clinical correlations, experimental manipulation of Treg levels in mouse models of atherosclerosis suggests a potentially causal relationship [91]. Furthermore, it has been suggested that a Treg-oriented immunomodulatory approach may offer therapeutic potential for atherosclerosis $[92,93]$.
The relationship between Treg dysfunction and atherosclerosis is further observed through the well-known incidence of atherosclerosis in various autoimmune diseases, most notably in the case of systemic lupus erythematosus (SLE) [94]. While the relationship between Tregs and SLE is complex, there is a general consensus that deficient Treg activity is one element of SLE [95], and thus, might also be a component of SLE-associated atherosclerosis [96]. Likewise, psoriasis and psoriatic arthritis, which are associated with Treg imbalances, have well-established associations with atherosclerosis [97-99]. The immune-CAD connection is seen quite clearly by an apparently causal relationship in immunemediated transplant arteriosclerosis [100]. Further evidence for the immune-CAD connection derives from the proven efficacy of rapamycin and related compounds, which are antibiotics/immunosuppressants, to block coronary artery restenosis. Rapamycin is known to increase Treg numbers and function at clinically relevant levels [101]. Recent findings provide fairly direct evidence that the cytokine responsiveness of $\mathrm{T}$ cell subsets is a better predictor of CAD than CRP [102]. Important recent studies indicate that Tregs license the pro-resolving abilities of plaque-resident macrophages in order to facilitate plaque regression [103].

Many of the TRACs identified herein have known relationships with Treg function, as shown schematically in Fig. 8. Foremost, the FoxP3 transcription factor is considered the definitive marker for the Treg subset and is

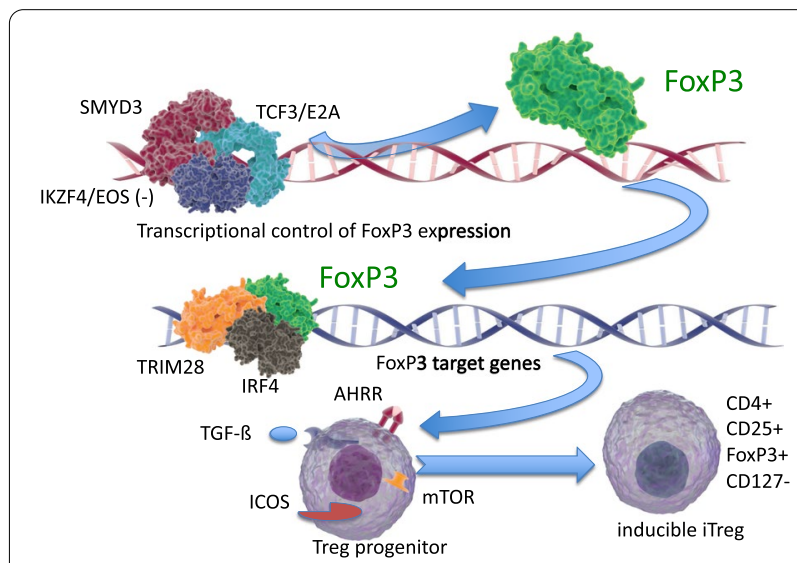

Fig. 8 Schematic representation of Treg-related TRACs identified by RNAseq. The control of FoxP3 mRNA and protein expression is known to be controlled by many factors, including promoter methylation, as well as transcriptional regulation by SMYD3, TCF3/E2A, and IKZF4/Eos. FoxP3, in turn, is itself a transcriptional regulator, in association with cofactors such as TRIM28, IRF4, and others. The FoxP3-sensitive target genes, and other regulators such as AHRR, ICOS, TGF- $B$, and mTOR, are then intrinsic components of the transition of Treg progenitor cells to functional Tregs. Molecular images courtesy of www.somersault 1824. com under a Creative Commons license 
thought to transcriptionally regulate a set of transcripts involved in Treg function. FoxP3 is itself epigenetically controlled by promoter demethylation and transcription factors, such as SMYD3, IKZF4/Eos, and TCF3/E2A, to allow stable expression in Tregs. Once transcribed and translated, FoxP3 regulates Treg-specific transcription via known promoter binding sites [55] and by interaction with a number of co-regulators, including RUNX1 [104], TRIM28, and IRF4 [105], which, along with SMYD3, IKZF4/Eos and TCF3, were identified as TRACs in the present studies. Other studies indicate that two isoforms of diacylglyceral kinase (DGKA, DGKZ) have been implicated in T-cell anergy [106], and DGKZ has been implicated in the generation of natural Tregs via modulation of the NFkB signaling through c-rel [107].

The ICOS/ICOSLG system is potentially important because athero-prone LDLR(-) mice transplanted with ICOS-deficient marrow develop more severe atherosclerosis [108]. Treg can be either ICOS+ or ICOS- [109], and typically ICOSLG is considered as a marker of a dendritic cell or innate lymphoid cell type 2 (ILC2), whereby ICOS on the Treg would engage ICOSLG on the ILC2s [110]. Flow cytometry analysis of Tregs in MI patients showed a subset of ICOS+ type to be preferentially affected [111]. Thus, the decrease in ICOSLG suggests that there may be some type of coordinate decrease in both Treg and ILC2 numbers or function.

The current studies differ from prior microarray-based analysis of CAD in several important ways. The current studies used a broader definition of CAD that would include earlier and more diffuse, but less occlusive disease. This definition also provided a better balance between the sizes of affected and unaffected groups. Further, prior studies used Paxgene blood RNA preservative, which is known to produce a very different RNA profile, while the present Tempus system was more sensitive for Treg markers. Likewise, RNAseq appears to be important for detecting changes in Treg activity and provides numerous quantitative and qualitative advantages over microarrays.

There are certain limitations in the present studies. First, the TRAC signature could be related to unidentified risk factors or drug treatments that differ between groups. While it is difficult to completely rule it out, based on the collected variables, we cannot identify a clinical covariate that would differ sufficiently to create this effect. Second, it is possible that the TRACs would detect disease in arteries other than the coronaries, but this would still have tremendous diagnostic value. A third limitation is that the clinical endpoint of an invasive coronary angiography (ICA) is excellent, but still imperfect at detecting coronary disease. Up to $75 \%$ of symptomatic patients that appear to have normal arteries by ICA can be shown by $\mathrm{CT}$ angiography to have significant atherosclerosis that does not occlude the artery [112]. The TRAC test would likely report these patients as positive, while they would be scored as angiographically negative for CAD by ICA. Future validation studies will need to incorporate $\mathrm{CT}$ angiography as an additional endpoint to avoid these ambiguous 'false positives' on blood-based tests. Likewise, TRAC-positive patients that are angiographically negative, could be in the early stages of the disease process, but that could be addressed only by a long-term follow-up study.

The present studies suggest several important directions for future investigation. Bioinformatically, it would be valuable to analyze the co-expression network of the transcripts, and analyze any RNA editing, differential splicing, and allele usage that might be occurring in CAD. The identification of RNA biomarkers that are associated with CAD has the potential to help dissect the mechanisms of atherosclerotic initiation and progression. It is likely worthwhile to further investigate the regulation of these transcripts by stress granules, as one component of immune dysfunction in coronary disease. Further, a potential connection between stress and the function of the immune synapse could elucidate specific mechanisms of disease, and targets for therapy, or prevention. Through high-throughput screening, dozens of FDAapproved compounds that stimulate Treg generation have already been identified [113]. Further refinement in the quantitation of these RNA biomarkers could lead to blood-based diagnostics for CAD, that would be a valuable addition to the diagnostic toolkit.

A long-term goal is to identify TRACs that may be predictive of CAD in asymptomatic, but 'at risk' individuals, especially middle age patients with one or more known risk factors [114]. Of the more than a million heart attacks per year in the US, approximately $50 \%$ of cases had no overt warning signs, and $50 \%$ of first heart attacks are fatal. Thus, an 'early warning sign' from blood-based RNA profiling could allow the patient to be referred for minimally-invasive diagnostics, such as stress tests, CT calcium scores, or MR/CT angiography, and thus hopefully reducing the incidence of heart attacks and strokes.

\section{Conclusions}

Transcriptome-wide profiling of whole blood RNA from patients with CAD identifies a pattern of changes that parallels known defects in the number and function of the regulatory $\mathrm{T}$ cell subset. The RNA pattern defines a risk that is independent of other known clinical risks, and thus could add value to future risk stratification models. Simple linear classification models using only seven transcripts provides surprisingly strong prediction of $\mathrm{CAD}$ status as determined by invasive 
coronary angiography. The RNA changes are consistent with stress-related changes in the immune synapse, which may help to define the precise cellular mechanisms of atherosclerotic lesion formation.

\begin{abstract}
Abbreviations
CAD: Coronary artery disease; CBC: Complete blood count; CPC: Circulating progenitor cells; ddPCR: Droplet digital PCR; DEGs: Differentially expressed genes; GERD: Gastroesophageal reflux disease; ICA: Invasive coronary angiography; PLSD: Partial least squares discriminant model; RIN: RNA integrity number; RNAseq: RNA sequencing; rRNA: Ribosomal RNA; RPKM: Reads per kilobase of exon per million mapped total reads; STEMI: ST segment elevation myocardial infarction; SG: Stress granules; SLE: Systemic lupus erythematosus; TRACs: Transcripts associated with CAD; Treg: Regulatory T cell.
\end{abstract}

\section{Supplementary Information}

The online version contains supplementary material available at https://doi. org/10.1186/s12920-021-01062-2.

Additional file 1. Six supplementary figures related to the RNAseq analysis of CAD.

Additional file 2. Ten supplementary tables providing transcript details for the RNAseq analysis of CAD.

\section{Acknowledgements}

The authors are grateful for the vision and determination of Dr. Georges St. Laurent, III, who sadly passed away during the final stages of this work. Georges was a genius, a friend, and a relentless force to help diagnose and treat human diseases.

\section{Authors' contributions}

G.S.L.3, R.K., I.T., and T.A.M. conceived and designed the studies. P.S., J.K., J.E., J.R., R.K., and R.M. identified and consented patients, collected clinical and laboratory data, and contributed clinical expertise on the conduct and analysis of the studies. Z.Y., I.T., M.T., D.J., D.S., T.J., and E.A., conducted RNA isolations, ddPCR, RNA sequencing and alignments. D.S., D.A., T.A.M., M.A., Z.A., Z.F., R.W., E.A., and Y.L. conducted the statistical, annotation, and bioinformatic analyses. T.A.M. wrote the manuscript with input from all the authors. All authors read and approved the final manuscript.

\section{Funding}

The authors are grateful for the financial support of the GW Heart and Vascular Institute, True Bearing Diagnostics, Inc. and The St. Laurent Institute, without which the studies would not have been possible. The authors are also grateful for the support of Award Number UL1TR001876 from the NIH National Center for Advancing Translational Sciences and Core Instrument Grant for the BioRad ddPCR S10 OD021622.

\section{Availability of data and materials}

The expression-level data (raw RPKM) is deposited in the Gene Expression Omnibus (GEO) at the accession \# GSE1 180083. The raw sequence files from this study will be provided to qualified investigators that can insure compliance with appropriate IRB and HIPPAA regulations for any future data usage, by contacting the corresponding author at mcc@gwu.edu. The human genome files for alignment were obtained from UCSC at this link for HG19 (https://hgdownload.soe.ucsc.edu/goldenPath/hg19/bigZips/) and HG38 (https://hgdownload.soe.ucsc.edu/goldenPath/hg38/bigZips/).

\section{Declarations}

\section{Ethics approval and consent to participate}

All subjects gave written, informed consent under IRB Protocol \#111015, approved by The George Washington University Institutional Review Board, and Protocol \# 15-2168 approved by the INOVA Fairfax IRB.

\section{Consent for publication}

Not applicable.

\section{Competing Interests}

TM, TJ, and IT have an equity interest in True Bearing Diagnostics, Inc., a diagnostics company developing RNA biomarkers for various diseases, including coronary artery disease. IT, GSL3, RK, and TM are seeking patent protection for a commercial diagnostic test, without restriction for research uses.

\section{Author details}

${ }^{1}$ Division of Genomic Medicine, Department of Medicine, The George Washington Medical Center, The George Washington University, 2300 I Street NW, Ross Hall 443A, Washington, DC 20037, USA. ${ }^{2}$ Division of Cardiology, Department of Medicine, The George Washington University, Washington, DC 20037, USA. ${ }^{3}$ SeqLL, Inc., Woburn, MA, USA. ${ }^{4}$ The St. Laurent Institute, Vancouver, WA, USA. ${ }^{5}$ Department of Statistics, Biostatistics Center, The George Washington University, Washington, DC 20037, USA. ${ }^{6}$ Department of Microbiology, Immunology, and Tropical Medicine, The George Washington University, Washington, DC 20037, USA. 'Department of Clinical Research and Leadership, The George Washington University, Washington, DC 20037, USA. ${ }^{8}$ A.P. Ershov Institute of Informatics Systems SB RAS, 6, Acad. Lavrentjeva Ave, Novosibirsk, Russia 630090. ${ }^{9}$ nnova Heart and Vascular Institute, Fairfax, VA, USA. ${ }^{10}$ True Bearing Diagnostics, Washington, DC 20037, USA.

Received: 9 May 2021 Accepted: 19 August 2021

Published online: 03 September 2021

\section{References}

1. Benjamin EJ, Blaha MJ, Chiuve SE, Cushman M, Das SR, Deo R, de Ferranti SD, Floyd J, Fornage M, Gillespie C, et al. Heart disease and stroke statistics-2017 update: A report from the American Heart Association. Circulation. 2017;135(10):e146-603.

2. Kohn MA, Kwan E, Gupta M, Tabas JA. Prevalence of acute myocardial infarction and other serious diagnoses in patients presenting to an urban emergency department with chest pain. J Emerg Med. 2005;29(4):383-90.

3. Klinkman MS, Stevens D, Gorenflo DW. Episodes of care for chest pain: a preliminary report from MIRNET. Michigan Research Network. J Fam Pract. 1994;38(4):345-52.

4. Pencina MJ, D’Agostino RB Sr, Larson MG, Massaro JM, Vasan RS. Predicting the 30-year risk of cardiovascular disease: the framingham heart study. Circulation. 2009;119(24):3078-84.

5. Patel MR, Peterson ED, Dai D, Brennan JM, Redberg RF, Anderson HV, Brindis RG, Douglas PS. Low diagnostic yield of elective coronary angiography. N Engl J Med. 2010;362(10):886-95.

6. Roger VL, Go AS, Lloyd-Jones DM, Adams RJ, Berry JD, Brown TM, Carnethon MR, Dai S, de Simone G, Ford ES, et al. Heart disease and stroke statistics-2011 update: a report from the American Heart Association. Circulation. 2010;6:66.

7. Farrehi PM, Bernstein SJ, Rasak M, Dabbous SA, Stomel RJ, Eagle KA, Rubenfire M. Frequency of negative coronary arteriographic findings in patients with chest pain is related to community practice patterns. Am J Manag Care. 2002;8(7):643-8.

8. Phibbs B, Fleming T, Ewy GA, Butman S, Ambrose J, Gorlin R, Orme $\mathrm{E}$, Mason J. Frequency of normal coronary arteriograms in three academic medical centers and one community hospital. Am J Cardiol. 1988;62(7):472-4.

9. Minha S, Behar S, Krakover R, Boyko V, Vered Z, Blatt A. Characteristics and outcome of patients with acute coronary syndrome and normal or near-normal coronary angiography. Coron Artery Dis. 2010;21(4):212-6.

10. From AM, Kane G, Bruce C, Pellikka PA, Scott C, McCully RB. Characteristics and outcomes of patients with abnormal stress echocardiograms and angiographically mild coronary artery disease $(<50 \%$ stenoses) or normal coronary arteries. J Am Soc Echocardiogr. 2010;23(2):207-14.

11. Rosenberg S, Elashoff MR, Beineke P, Daniels SE, Wingrove JA, Tingley WG, Sager PT, Sehnert AJ, Yau M, Kraus WE, et al. Multicenter validation of the diagnostic accuracy of a blood-based gene expression test for assessing obstructive coronary artery disease in nondiabetic patients. Ann Intern Med. 2010;153(7):425-34. 
12. Elashoff MR, Wingrove JA, Beineke P, Daniels SE, Tingley WG, Rosenberg S, Voros S, Kraus WE, Ginsburg GS, Schwartz RS, et al. Development of a blood-based gene expression algorithm for assessment of obstructive coronary artery disease in non-diabetic patients. BMC Med Genomics. 2011;4:26.

13. Huan $T$, Zhang $B$, Wang $Z$, Joehanes $R$, Zhu J, Johnson AD, Ying $S$, Munson PJ, Raghavachari N, Wang R, et al. A systems biology framework identifies molecular underpinnings of coronary heart disease. Arterioscler Thromb Vasc Biol. 2013:33(6):1427-34.

14. Sinnaeve PR, Donahue MP, Grass P, Seo D, Vonderscher J, Chibout SD, Kraus WE, Sketch M Jr, Nelson C, Ginsburg GS, et al. Gene expression patterns in peripheral blood correlate with the extent of coronary artery disease. PLoS ONE. 2009;4(9):e7037.

15. Wingrove JA, Daniels SE, Sehnert AJ, Tingley W, Elashoff MR, Rosenberg S, Buellesfeld L, Grube E, Newby LK, Ginsburg GS, et al. Correlation of peripheral-blood gene expression with the extent of coronary artery stenosis. Circ Cardiovasc Genet. 2008;1(1):31-8.

16. Li L, Ying L, Naesens M, Xiao W, Sigdel T, Hsieh S, Martin J, Chen R, Liu $K$, Mindrinos $M$, et al. Interference of globin genes with biomarker discovery for allograft rejection in peripheral blood samples. Physiol Genomics. 2008;32(2):190-7.

17. Goff DC Jr, Lloyd-Jones DM, Bennett G, Coady S, D'Agostino RB, Gibbons R, Greenland P, Lackland DT, Levy D, O'Donnell CJ, et al. 2013 ACC/ AHA guideline on the assessment of cardiovascular risk: a report of the American College of Cardiology/American Heart Association Task Force on Practice Guidelines. Circulation. 2014;129(25 Suppl 2):S49-73.

18. Min JK, Dunning A, Gransar H, Achenbach S, Lin FY, Al-Mallah M, Budoff MJ, Callister TQ, Chang HJ, Cademartiri F, et al. Medical history for prognostic risk assessment and diagnosis of stable patients with suspected coronary artery disease. Am J Med. 2015;6:66.

19. Giladi E, Healy J, Myers G, Hart C, Kapranov P, Lipson D, Roels S, Thayer E, Letovsky S. Error tolerant indexing and alignment of short reads with covering template families. J Comput Biol. 2010;17(10):1397-411.

20. Menke A, Rex-Haffner M, Klengel T, Binder EB, Mehta D. Peripheral blood gene expression: it all boils down to the RNA collection tubes. BMC Res Notes. 2012;5:1.

21. Shi L, Reid LH, Jones WD, Shippy R, Warrington JA, Baker SC, Collins PJ, de Longueville F, Kawasaki ES, Lee KY, et al. The MicroArray Quality Control (MAQC) project shows inter- and intraplatform reproducibility of gene expression measurements. Nat Biotechnol. 2006;24(9):1151-61.

22. Ladapo JA, Budoff M, Sharp D, Zapien M, Huang L, Maniet B, Herman $\mathrm{L}$, Monane M. Clinical utility of a precision medicine test evaluating outpatients with suspected obstructive coronary artery disease. Am J Med. 2016;6:66

23. ROC Analysis: web-based calculator for ROC curves. http://www.jrocfit. org.

24. Larson NB, Berardi C, Decker PA, Wassel CL, Kirsch PS, Pankow JS, Sale MM, de Andrade M, Sicotte H, Tang W, et al. Trans-ethnic meta-analysis identifies common and rare variants associated with hepatocyte growth factor levels in the Multi-Ethnic Study of Atherosclerosis (MESA). Ann Hum Genet. 2015;79(4):264-74.

25. McDonough CW, Gong Y, Padmanabhan S, Burkley B, Langaee TY, Melander O, Pepine CJ, Dominiczak AF, Cooper-Dehoff RM, Johnson JA. Pharmacogenomic association of nonsynonymous SNPs in SIGLEC12, $\mathrm{A} 1 \mathrm{BG}$, and the selectin region and cardiovascular outcomes. Hypertension. 2013;62(1):48-54.

26. Lu X, Wang L, Chen S, He L, Yang X, Shi Y, Cheng J, Zhang L, Gu CC, Huang J, et al. Genome-wide association study in Han Chinese identifies four new susceptibility loci for coronary artery disease. Nat Genet. 2012;44(8):890-4.

27. Choi JR, Jang Y, Kim Yoon S, Park JK, Sorn SR, Park MY, Lee M. The impact of $\mathrm{CDH} 13$ polymorphism and statin administration on TG/HDL ratio in cardiovascular patients. Yonsei Med J. 2015;56(6):1604-12.

28. Putku M, Kals M, Inno R, Kasela S, Org E, Kozich V, Milani L, Laan M. CDH13 promoter SNPs with pleiotropic effect on cardiometabolic parameters represent methylation QTLs. Hum Genet. 2015;134(3):291-303.

29. Solban N, Dumas P, Gossard F, Sun Y, Pravenec M, Kren V, Lewanczuk $R$, Hamet $P$, Tremblay J. Chromosomal mapping of HCaRG, a novel hypertension-related, calcium-regulated gene. Folia Biol (Praha). 2002;48(1):9-14.
30. Park YM, Province MA, Gao X, Feitosa M, Wu J, Ma D, Rao D, Kraja AT. Longitudinal trends in the association of metabolic syndrome with $550 \mathrm{k}$ single-nucleotide polymorphisms in the Framingham Heart Study. BMC Proc. 2009;3(Suppl 7):S116.

31. Lessard CJ, Sajuthi S, Zhao J, Kim K, Ice JA, Li H, Ainsworth H, Rasmussen A, Kelly JA, Marion M, et al. Identification of a systemic lupus erythematosus risk locus spanning ATG16L2, FCHSD2, and P2RY2 in Koreans. Arthritis Rheumatol. 2016;68(5):1197-209.

32. Hubacek JA, Stanek V, Gebauerova M, Poledne R, Aschermann M, Skalicka H, Matouskova J, Kruger A, Penicka M, Hrabakova H, et al. Rs6922269 marker at the MTHFD1L gene predict cardiovascular mortality in males after acute coronary syndrome. Mol Biol Rep. 2015:42(8):1289-93.

33. Saade $S$, Cazier JB, Ghassibe-Sabbagh M, Youhanna S, Badro DA, Kamatani Y, Hager J, Yeretzian JS, El-Khazen G, Haber M, et al. Large scale association analysis identifies three susceptibility loci for coronary artery disease. PLOS ONE. 2011;6(12):e29427.

34. Guardiola M, Exeter HJ, Perret C, Folkersen L, Van't Hooft F, Eriksson P, Franco-Cereceda A, Paulsson-Berne G, Palmen J, Li K, et al. PLA2G10 gene variants, sPLA2 activity, and coronary heart disease risk. Circ Cardiovasc Genet. 2015;8(2):356-62.

35. Ait-Oufella H, Herbin O, Lahoute C, Coatrieux C, Loyer X, Joffre J, Laurans L, Ramkhelawon B, Blanc-Brude O, Karabina S, et al. Group $X$ secreted phospholipase A2 limits the development of atherosclerosis in LDL receptor-null mice. Arterioscler Thromb Vasc Biol. 2013;33(3):466-73.

36. Clop A, Bertoni A, Spain SL, Simpson MA, Pullabhatla V, Tonda R, Hundhausen C, Di Meglio P, De Jong P, Hayday AC, et al. An in-depth characterization of the major psoriasis susceptibility locus identifies candidate susceptibility alleles within an HLA-C enhancer element. PLOS ONE. 2013:8(8):e71690.

37. Sun $H$, Xia Y, Wang L, Wang Y, Chang X. PSORS1C1 may be involved in rheumatoid arthritis. Immunol Lett. 2013;153(1-2):9-14.

38. Xie Z, Nagarajan V, Sturdevant DE, Iwaki S, Chan E, Wisch L, Young M, Nelson CM, Porcella SF, Druey KM. Genome-wide SNP analysis of the systemic capillary leak syndrome (Clarkson disease). Rare Dis. 2013;1(1):66.

39. Iotchkova V, Huang J, Morris JA, Jain D, Barbieri C, Walter K, Min JL, Chen L, Astle W, Cocca M, et al. Discovery and refinement of genetic loci associated with cardiometabolic risk using dense imputation maps. Nat Genet. 2016;6:66.

40. Lu H, Guo P. Plasma heparin cofactor II activity correlates with the incidence of in-stent restenosis after the intervention of arteriosclerosis obliterans in lower extremity. Zhong Nan Da Xue Xue Bao Yi Xue Ban. 2015;40(2):177-81.

41. Rau JC, Deans C, Hoffman MR, Thomas DB, Malcom GT, Zieske AW, Strong JP, Koch GG, Church FC. Heparin cofactor II in atherosclerotic lesions from the pathobiological determinants of atherosclerosis in youth (PDAY) study. Exp Mol Pathol. 2009;87(3):178-83.

42. Foroughi Asl H, Talukdar HA, Kindt AS, Jain RK, Ermel R, Ruusalepp A, Nguyen KD, Dobrin R, Reilly DF, Schunkert H, et al. Expression quantitative trait Loci acting across multiple tissues are enriched in inherited risk for coronary artery disease. Circ Cardiovasc Genet. 2015;8(2):305-15.

43. Thomsen SK, Ceroni A, van de Bunt M, Burrows C, Barrett A, Scharfmann R, Ebner D, McCarthy MI, Gloyn AL. Systematic functional characterization of candidate causal genes for type 2 diabetes risk variants. Diabetes. 2016;6:66

44. Chen Q, Coffey A, Bourgoin SG, Gadina M. Cytohesin binder and regulator augments $T$ cell receptor-induced nuclear factor of activated T cells. AP-1 activation through regulation of the JNK pathway. J Biol Chem. 2006;281(29):19985-94.

45. Wang L, Ni X, Covington KR, Yang BY, Shiu J, Zhang X, Xi L, Meng Q, Langridge T, Drummond J, et al. Genomic profiling of Sezary syndrome identifies alterations of key $T$ cell signaling and differentiation genes. Nat Genet. 2015;47(12):1426-34.

46. Silva O, Crocetti J, Humphries LA, Burkhardt JK, Miceli MC. Discs large homolog 1 splice variants regulate p38-dependent and -independent effector functions in CD8+ T Cells. PLoS ONE. 2015;10(7):e0133353. 
47. Choi JM, Bothwell AL. The nuclear receptor PPARs as important regulators of T-cell functions and autoimmune diseases. Mol Cells. 2012;33(3):217-22.

48. Koreishi AF, Saenz AJ, Persky DO, Cui H, Moskowitz A, Moskowitz $\mathrm{CH}$, Teruya-Feldstein J. The role of cytotoxic and regulatory T cells in relapsed/refractory Hodgkin lymphoma. Appl Immunohistochem Mol Morphol. 2010;18(3):206-11.

49. Schmidt A, Zhang XM, Joshi RN, lqbal S, Wahlund C, Gabrielsson S, Harris RA, Tegner J. Human macrophages induce CD4(+)Foxp3(+) regulatory $T$ cells via binding and re-release of TGF-beta. Immunol Cell Biol. 2016;94(8):747-62.

50. Sharma MD, Huang L, Choi JH, Lee EJ, Wilson JM, Lemos H, Pan F, Blazar $B R$, Pardoll DM, Mellor AL, et al. An inherently bifunctional subset of Foxp3+ Thelper cells is controlled by the transcription factor eos. Immunity. 2013;38(5):998-1012.

51. Pan F, Yu H, Dang EV, Barbi J, Pan X, Grosso JF, Jinasena D, Sharma SM, McCadden EM, Getnet D, et al. Eos mediates Foxp3-dependent gene silencing in CD4+ regulatory T cells. Science. 2009;325(5944):1142-6.

52. Nagata DE, Ting HA, Cavassani KA, Schaller MA, Mukherjee S, Ptaschinski C, Kunkel SL, Lukacs NW. Epigenetic control of Foxp3 by SMYD3 H3K4 histone methyltransferase controls iTreg development and regulates pathogenic T-cell responses during pulmonary viral infection. Mucosal Immunol. 2015;8(5):1131-43.

53. Maruyama T, Li J, Vaque JP, Konkel JE, Wang W, Zhang B, Zhang P, Zamarron BF, Yu D, Wu Y, et al. Control of the differentiation of regulatory $T$ cells and $T(H) 17$ cells by the DNA-binding inhibitor Id3. Nat Immunol. 2011:12(1):86-95.

54. Huang C, Martin S, Pfleger C, Du J, Buckner JH, Bluestone JA, Riley JL, Ziegler SF. Cutting Edge: a novel, human-specific interacting protein couples FOXP3 to a chromatin-remodeling complex that contains KAP1/TRIM28. J Immunol. 2013;190(9):4470-3.

55. Huehn J, Beyer M. Epigenetic and transcriptional control of Foxp3+ regulatory T cells. Semin Immunol. 2015;27(1):10-8.

56. Palmer C, Diehn M, Alizadeh AA, Brown PO. Cell-type specific gene expression profiles of leukocytes in human peripheral blood. BMC Genomics. 2006;7:115.

57. Wojakowski W, Landmesser U, Bachowski R, Jadczyk T, Tendera M. Mobilization of stem and progenitor cells in cardiovascular diseases. Leuk Off J Leuk Soc Am Leuk Res Fund UK. 2012;26(1):23-33.

58. Fadini GP, Maruyama S, Ozaki T, Taguchi A, Meigs J, Dimmeler S, Zeiher AM, de Kreutzenberg S, Avogaro A, Nickenig G, et al. Circulating progenitor cell count for cardiovascular risk stratification: a pooled analysis. PLOS ONE. 2010;5(7):11488.

59. Werner N, Kosiol S, SchiegI T, Ahlers P, Walenta K, Link A, Bohm M, Nickenig G. Circulating endothelial progenitor cells and cardiovascular outcomes. N Engl J Med. 2005;353(10):999-1007.

60. Chironi G, Walch L, Pernollet MG, Gariepy J, Levenson J, Rendu F, Simon A. Decreased number of circulating CD34+KDR + cells in asymptomatic subjects with preclinical atherosclerosis. Atherosclerosis. 2007;191(1):115-20

61. Vasa M, Fichtlscherer S, Aicher A, Adler K, Urbich C, Martin H, Zeiher AM, Dimmeler S. Number and migratory activity of circulating endothelial progenitor cells inversely correlate with risk factors for coronary artery disease. Circ Res. 2001;89(1):E1-7.

62. Damani S, Bacconi A, Libiger O, Chourasia AH, Serry R, Gollapudi R, Goldberg R, Rapeport K, Haaser S, Topol S, et al. Characterization of circulating endothelial cells in acute myocardial infarction. Sci Transl Med. 2012;4(126):126-33.

63. George J, Schwartzenberg S, Medvedovsky D, Jonas M, Charach G, Afek A, Shamiss A. Regulatory T cells and IL-10 levels are reduced in patients with vulnerable coronary plaques. Atherosclerosis. 2012;222(2):519-23.

64. Li Q. Peripheral Th17/Treg imbalance in patients with atherosclerotic cerebral infarction. Int J Clin Exp Pathol. 2013;6(6):1015-27.

65. Asare AL, Kolchinsky SA, Gao Z, Wang R, Raddassi K, Bourcier K, SeyfertMargolis V. Differential gene expression profiles are dependent upon method of peripheral blood collection and RNA isolation. BMC Genomics. 2008;9:474.

66. Matheson LA, Duong TT, Rosenberg AM, Yeung RS. Assessment of sample collection and storage methods for multicenter immunologic research in children. J Immunol Methods. 2008;339(1):82-9.
67. Ren J, Han L, Tang J, Liu Y, Deng X, Liu Q, Hao P, Feng X, Li B, Hu H, et al. Foxp1 is critical for the maintenance of regulatory $T$-cell homeostasis and suppressive function. PLoS Biol. 2019;17(5):e3000270.

68. Namkoong S, Ho A, Woo YM, Kwak H, Lee JH. Systematic characterization of stress-induced RNA granulation. Mol Cell. 2018;70(1):175e178-187e178.

69. Taschuk F, Cherry S. DEAD-Box helicases: sensors, regulators, and effectors for antiviral defense. Viruses. 2020;12(2):66.

70. Weil D, Piton A, Lessel D, Standart N. Mutations in genes encoding regulators of mRNA decapping and translation initiation: links to intellectual disability. Biochem Soc Trans. 2020;48(3):1199-211.

71. Vindry C, Weil D, Standart N. Pat1 RNA-binding proteins: multitasking shuttling proteins. Wiley Interdiscip Rev RNA. 2019;10(6):e1557.

72. Eriksson M, Brown WT, Gordon LB, Glynn MW, Singer J, Scott L, Erdos MR, Robbins CM, Moses TY, Berglund P, et al. Recurrent de novo point mutations in lamin A cause Hutchinson-Gilford progeria syndrome. Nature. 2003;423(6937):293-8.

73. Nussing S, Koay HF, Sant S, Loudovaris T, Mannering SI, Lappas M, Du Y, Konstantinov IE, Berzins SP, Rimmelzwaan GF, et al. Divergent SATB1 expression across human life span and tissue compartments. Immunol Cell Biol. 2019;97(5):498-511.

74. Akimova T, Zhang T, Negorev D, Singhal S, Stadanlick J, Rao A, Annunziata M, Levine MH, Beier UH, Diamond JM, et al. Human lung tumor FOXP3+ Tregs upregulate four "Treg-locking" transcription factors. JCI Insight. 2017;2(16):66

75. Hsu Y, Garrison JE, Seo S, Sheffield VC. The absence of BBSome function decreases synaptogenesis and causes ectopic synapse formation in the retina. Sci Rep. 2020;10(1):8321.

76. van der Harst P, Verweij N. Identification of 64 novel genetic loci provides an expanded view on the genetic architecture of coronary artery disease. Circ Res. 2018;122(3):433-43.

77. Tan B, Liu L, Yang Y, Liu Q, Yang L, Meng F. Low CPNE3 expression is associated with risk of acute myocardial infarction: a feasible genetic marker of acute myocardial infarction in patients with stable coronary artery disease. Cardiol J. 2019;26(2):186-93.

78. Allam AH, Charnley M, Pham K, Russell SM. Developing T cells form an immunological synapse for passage through the beta-selection checkpoint. J Cell Biol. 2021;220(3):66.

79. Ross R. The pathogenesis of atherosclerosis: a perspective for the 1990s. Nature. 1993;362:801-9.

80. Hansson GK. Immune mechanisms in atherosclerosis. Arterioscler Thromb Vasc Biol. 2001;21(12):1876-90.

81. Libby P, Okamoto Y, Rocha VZ, Folco E. Inflammation in atherosclerosis: transition from theory to practice. Circ J. 2010;74(2):213-20.

82. Depuydt MAC, Prange KHM, Slenders L, Ord T, Elbersen D, Boltjes A, de Jager SCA, Asselbergs FW, de Borst GJ, Aavik E, et al. Microanatomy of the human atherosclerotic plaque by single-cell transcriptomics. Circ Res. 2020;127(11):1437-55.

83. Ketelhuth DF, Hansson GK. Modulation of autoimmunity and atherosclerosis-common targets and promising translational approaches against disease. Circ J. 2015;79(5):924-33.

84. Cheng X, Yu X, Ding YJ, Fu QQ, Xie JJ, Tang TT, Yao R, Chen Y, Liao YH. The Th17/Treg imbalance in patients with acute coronary syndrome. Clin Immunol. 2008;127(1):89-97.

85. Liu M, Xu LJ, Wu JX. Changes of circulating CD4(+)CD25(+)CD127(low) regulatory $T$ cells in patients with acute coronary syndrome and its significance. Genet Mol Res. 2015;14(4):15930-6.

86. Liu ZD, Wang L, Lu FH, Pan H, Zhao YX, Wang SJ, Sun SW, Li CL, Hu XL. Increased Th17 cell frequency concomitant with decreased Foxp3+ Treg cell frequency in the peripheral circulation of patients with carotid artery plaques. Inflamm Res. 2012;61(10):1155-65.

87. Emoto T, Sasaki N, Yamashita T, Kasahara K, Yodoi K, Sasaki Y, Matsumoto T, Mizoguchi T, Hirata K. Regulatory/effector T-cell ratio is reduced in coronary artery disease. Circ J. 2014;78(12):2935-41.

88. Potekhina AV, Pylaeva E, Provatorov S, Ruleva N, Masenko V, Noeva E, Krasnikova T, Arefieva T. Treg/Th17 balance in stable CAD patients with different stages of coronary atherosclerosis. Atherosclerosis. 2015;238(1):17-21.

89. Kim YC, Kim KK, Shevach EM. Simvastatin induces Foxp3+ T regulatory cells by modulation of transforming growth factor-beta signal transduction. Immunology. 2010;130(4):484-93. 
90. Rodriguez-Perea AL, Montoya CJ, Olek S, Chougnet CA, Velilla PA. Statins increase the frequency of circulating CD4+ FOXP3+ regulatory $T$ cells in healthy individuals. J Immunol Res. 2015;2015:762506.

91. Ait-Oufella H, Salomon BL, Potteaux S, Robertson AK, Gourdy P, Zoll J, Merval R, Esposito B, Cohen JL, Fisson S, et al. Natural regulatory $T$ cells control the development of atherosclerosis in mice. Nat Med. 2006:12(2):178-80.

92. Yamashita T, Sasaki N, Kasahara K, Hirata K. Anti-inflammatory and immune-modulatory therapies for preventing atherosclerotic cardiovascular disease. J Cardiol. 2015;66(1):1-8.

93. Spitz C, Winkels H, Burger C, Weber C, Lutgens E, Hansson GK, Gerdes $\mathrm{N}$. Regulatory $T$ cells in atherosclerosis: critical immune regulatory function and therapeutic potential. Cell Mol Life Sci. 2015;6:66.

94. Roldan PC, Ratliff M, Snider R, Macias L, Rodriguez R, Sibbitt W, Roldan CA. Aortic atherosclerosis in systemic lupus erythematosus. Rheumatology. 2014;5:66.

95. Ohl K, Tenbrock K. Regulatory T cells in systemic lupus erythematosus. Eur J Immunol. 2015;45(2):344-55.

96. Zhu M, Mo H, Li D, Luo X, Zhang L. Th17/Treg imbalance induced by increased incidence of atherosclerosis in patients with systemic lupus erythematosus (SLE). Clin Rheumatol. 2013;32(7):1045-52.

97. Teague H, Mehta NN. The link between inflammatory disorders and coronary heart disease: a look at recent studies and novel drugs in development. Curr Atheroscler Rep. 2016:18(1):3.

98. Yilmazer B, Sahin T, Unlu BO, Kir HM, Cefle A. Investigation of subclinical atherosclerosis in psoriatic arthritis patients with minimal disease activity. Rheumatol Int. 2015;35(8):1385-92.

99. Armstrong AW, Voyles SV, Armstrong EJ, Fuller EN, Rutledge JC. A tale of two plaques: convergent mechanisms of T-cell-mediated inflammation in psoriasis and atherosclerosis. Exp Dermatol. 2011;20(7):544-9.

100. von Rossum A, Laher I, Choy JC. Immune-mediated vascular injury and dysfunction in transplant arteriosclerosis. Front Immunol. 2014;5:684.

101. Fanigliulo D, Lazzerini $P E$, Capecchi PL, Ulivieri C, Baldari CT, LaghiPasini F. Clinically-relevant cyclosporin and rapamycin concentrations enhance regulatory $T$ cell function to a similar extent but with different mechanisms: an in-vitro study in healthy humans. Int Immunopharmacol. 2015;24(2):276-84.

102. Shen-Orr SS, Furman D, Kidd BA, Hadad F, Lovelace P, Huang YW, Rosenberg-Hasson Y, Mackey S, Grisar FA, Pickman Y, et al. Defective signaling in the JAK-STAT pathway tracks with chronic inflammation and cardiovascular risk in aging humans. Cell Syst. 2016;3(4):374e374-384e374.

103. Sharma M, Schlegel MP, Afonso MS, Brown EJ, Rahman K, Weinstock A Sansbury BE, Corr EM, van Solingen C, Koelwyn GJ, et al. Regulatory T cells license macrophage pro-resolving functions during atherosclerosis regression. Circ Res. 2020;127(3):335-53.

104. Ono M, Yaguchi H, Ohkura N, Kitabayashi I, Nagamura Y, Nomura T, Miyachi Y, Tsukada T, Sakaguchi S. Foxp3 controls regulatory T-cell function by interacting with AML1/Runx1. Nature. 2007:446(7136):685-9.

105. Zheng Y, Chaudhry A, Kas A, deRoos P, Kim JM, Chu TT, Corcoran L, Treuting P, Klein U, Rudensky AY. Regulatory T-cell suppressor program co-opts transcription factor IRF4 to control T(H)2 responses. Nature. 2009;458(7236):351-6.

106. Zhong XP, Guo R, Zhou H, Liu C, Wan CK. Diacylglycerol kinases in immune cell function and self-tolerance. Immunol Rev. 2008:224:249-64.

107. Schmidt AM, Zou T, Joshi RP, Leichner TM, Pimentel MA, Sommers CL, Kambayashi T. Diacylglycerol kinase zeta limits the generation of natural regulatory T cells. Sci Signal. 2013;6(303):ra101.

108. Gotsman I, Grabie N, Gupta R, Dacosta R, MacConmara M, Lederer J, Sukhova G, Witztum JL, Sharpe AH, Lichtman AH. Impaired regulatory T-cell response and enhanced atherosclerosis in the absence of inducible costimulatory molecule. Circulation. 2006;114(19):2047-55.

109. Zhang S, Yong LK, Li D, Cubas R, Chen C, Yao Q. Mesothelin virus-like particle immunization controls pancreatic cancer growth through CD8+ T cell induction and reduction in the frequency of CD4+ foxp3+ ICOS - regulatory T cells. PloS ONE. 2013;8(7):e68303.

110. Rigas D, Lewis G, Aron JL, Wang B, Banie H, Sankaranarayanan I, GalleTreger L, Maazi H, Lo R, Freeman GJ, et al. ILC2 suppression by regulatory $T$ cells attenuates airway hyperreactivity and requires ICOS:ICOSligand interaction. J Allergy Clin Immunol. 2016;6:66.

111. Ghourbani Gazar S, Andalib A, Hashemi M, Rezaei A. CD4(+)Foxp3(+) Treg and its ICOS(+) subsets in patients with myocardial infarction. Iran J Immunol. 2012;9(1):53-60.

112. Bittner DO, Klinghammer L, Marwan M, Schmid J, Layritz C, Hoffmann U, Achenbach S, Pflederer T. Influence of cardiovascular risk factors on the prevalence of coronary atherosclerosis in patients with angiographically normal coronary arteries. Acad Radiol. 2017;24(5):580-6.

113. Mao R, Xiao W, Liu H, Chen B, Yi B, Kraj P, She JX. Systematic evaluation of 640 FDA drugs for their effect on CD4(+)Foxp3(+) regulatory T cells using a novel cell-based high throughput screening assay. Biochem Pharmacol. 2013:85(10):1513-24.

114. Weintraub WS, Daniels SR, Burke LE, Franklin BA, Goff DC Jr, Hayman LL, Lloyd-Jones D, Pandey DK, Sanchez EJ, Schram AP, et al. Value of primordial and primary prevention for cardiovascular disease: a policy statement from the American Heart Association. Circulation. 2011;124(8):967-90.

\section{Publisher's Note The style of headers below seem} to change randomly, is that correct?

Springer Nature remains neutral with regard to jurisdictional claims in published maps and institutional affiliations.

Ready to submit your research? Choose BMC and benefit from:

- fast, convenient online submission

- thorough peer review by experienced researchers in your field

- rapid publication on acceptance

- support for research data, including large and complex data types

- gold Open Access which fosters wider collaboration and increased citations

- maximum visibility for your research: over 100M website views per year

At BMC, research is always in progress.

Learn more biomedcentral.com/submissions 